\title{
Exact Computable Representation of Some Second-Order Cone Constrained Quadratic Programming Problems
}

\author{
Qingwei Jin • Ye Tian • Zhibin Deng • \\ Shu-Cherng Fang • Wenxun Xing
}

Received: 16 December 2012 / Revised: 26 February 2013 / Accepted: 2 March 2013 /

Published online: 20 March 2013

(C) Operations Research Society of China, Periodicals Agency of Shanghai University, and

Springer-Verlag Berlin Heidelberg 2013

\begin{abstract}
Solving the quadratically constrained quadratic programming (QCQP) problem is in general NP-hard. Only a few subclasses of the QCQP problem are known to be polynomial-time solvable. Recently, the QCQP problem with a nonconvex quadratic objective function over one ball and two parallel linear constraints is proven to have an exact computable representation, which reformulates the original problem as a linear semidefinite program with additional linear and second-order
\end{abstract}

This work was generously supported by US Army Research Office Grant (No. W911NF-04-D-0003), by the North Carolina State University Edward P. Fitts Fellowship and by National Natural Science Foundation of China (No. 11171177). It is the policy of the Army Research Office that university personnel do not need to do joint work with ARO personnel in order to receive grants from the Army Research Office.

Q. Jin

Department of Management Science and Engineering, Zhejiang University, Hangzhou, 310058,

China

e-mail: qingweijin@zju.edu.cn

Y. Tian $(\bowtie)$

School of Business Administration, Southwestern University of Finance and Economics, Chengdu, 611130, China

e-mail: tianye7272@gmail.com

Z. Deng · S.-C. Fang

Edward P. Fitts Department of Industrial and Systems Engineering, North Carolina State University, Raleigh, NC 27695, USA

Z. Deng

e-mail: zdeng2@ncsu.edu

S.-C. Fang

e-mail: fang@ncsu.edu

W. Xing

Department of Mathematical Sciences, Tsinghua University, Beijing, 100084, China

e-mail: wxing@math.tsinghua.edu.cn 
cone constraints. In this paper, we provide exact computable representations for some more subclasses of the QCQP problem, in particular, the subclass with one secondorder cone constraint and two special linear constraints.

Keywords Linear conic program - Semidefinite program · Nonconvex quadratically constrained quadratic program $\cdot$ Second-order cone

\section{Introduction}

The quadratically constrained quadratic programming (QCQP) problem can be expressed as

$$
\begin{aligned}
& \inf x^{T} A_{0} x+2 b_{0}^{T} x+c_{0} \\
& \text { s.t. } \quad x \in \mathcal{F}
\end{aligned}
$$

where the feasible domain $\mathcal{F} \triangleq\left\{x \in \mathbb{R}^{n} \mid x^{T} A_{i} x+2 b_{i}^{T} x+c_{i} \leqslant 0, i=1, \cdots, m_{1}\right.$, $\left.x^{T} A_{j} x+2 b_{j}^{T} x+c_{j}=0, j=m_{1}+1, \cdots, m_{1}+m_{2}\right\}$ with $A_{i}, A_{j} \in \mathcal{S}^{n}$, the space of real symmetric square matrices of order $n, b_{i}, b_{j} \in \mathbb{R}^{n}$, the $n$-dimensional real space, and $c_{i}, c_{j} \in \mathbb{R}, i=0,1, \cdots, m_{1}, j=m_{1}+1, \cdots, m_{1}+m_{2}$. This problem has been extensively studied and proven to be NP-hard even if all of the constraints are linear (Ref. [10]). For the convex QCQP problem, it can be reformulated as a linear second-order cone programming problem and then solved in polynomial time using interior point methods (Ref. [9]). For the nonconvex QCQP problem, only some subclasses are known to be computable. Here, "computable" means a problem can be solved within an arbitrary precision level in polynomial time. In the literature, linear constraints, second-order cone constraints and semidefinite constraints are commonly used to construct an equivalent representation of a given QCQP problem. When the equivalent problem is polynomial-time solvable and the size of such a representation is polynomial in terms of the size of the original problem, then we say it is a "computable representation." Computable representations of QCQP with $\mathcal{F}$ being defined by one nonconvex quadratic inequality constraint, or by one strictly convex/concave quadratic equality constraint, or by one convex quadratic inequality and one linear inequality can be found in Sturm and Zhang [13]. Moreover, the computable representation in [13] also works for the QCQP with $\mathcal{F}$ being defined by two convex quadratic inequality constraints sharing the same Hessian matrix. Kim and Kojima [7] proposed a semidefinite representation and a second-order cone representation for QCQP problems whose matrix formulations have coefficients being uniformly almost OD-nonpositive. (A real symmetric matrix is OD-nonpositive if its off-diagonal elements are nonpositive.) Furthermore, Ye and Zhang [14] provided a semidefinite representation for three subclasses of the QCQP problem with two quadratic constraints: (i) one of the two constraints in the SDP relaxation is not binding, (ii) the two constraints and the objective function are all in the homogeneous form, and (iii) one is an elliptic constraint and the other is a linear complementarity constraint. Recently, Burer and Anstreicher [2] showed an exact computable representation of QCQP with one elliptic constraint and two parallel linear constraints. However, the computable representation of QCQP problems with two binding elliptic constraints 
or one second-order cone constraint is still unknown. (Note that having a secondorder cone constraint is equivalent to having one quadratic constraint and one linear constraint, not merely one quadratic constraint.)

In this paper, we will show computable representations of QCQP problems with the following feasible domains:

- $\mathcal{F}=\left\{(x, y) \in \mathbb{R}^{n_{1}} \times \mathbb{R}^{n_{2}} \mid\|x\| \leqslant a_{1}+a_{2}^{T} x+a_{3}^{T} y, a_{1}+a_{2}^{T} x+a_{3}^{T} y \geqslant a_{4} \geqslant 0\right\}$ with $a_{1} \in \mathbb{R}, a_{2} \in \mathbb{R}^{n_{1}}, a_{3} \in \mathbb{R}^{n_{2}}$ and $a_{4} \geqslant 0$.

- $\mathcal{F}=\left\{(x, y) \in \mathbb{R}^{n_{1}} \times \mathbb{R}^{n_{2}} \mid\|x\| \leqslant a_{1}+a_{2}^{T} x+a_{3}^{T} y, a_{5} \geqslant a_{1}+a_{2}^{T} x+a_{3}^{T} y \geqslant a_{4} \geqslant 0\right\}$ with $a_{1} \in \mathbb{R}, a_{2} \in \mathbb{R}^{n_{1}}, a_{3} \in \mathbb{R}^{n_{2}}$ and $a_{5}>a_{4} \geqslant 0$.

The above representations generalize the ball constraint and the second-order cone constraint. As a corollary, one can obtain the computable representation of the widely used second-order cone constraint $c^{T} x+d \geqslant\|A x+b\|$ with $l \leqslant c^{T} x+d \leqslant u$, in which $c \in \mathbb{R}^{n}, d, l, u \in \mathbb{R}, A \in \mathbb{R}^{m \times n}$ and $b \in \mathbb{R}^{m}$. In particularly, when $\mathcal{F}=$ $\left\{\left(x_{0}, x\right) \in \mathbb{R} \times \mathbb{R}^{n} \mid\|x\| \leqslant x_{0}\right\}$, the computable representation derived in this paper answers the question in Proposition 8.7 of [3]. Another motivation is that, in [4], a QCQP problem can be reformulated as a QCQP problem over an intersection of several second-order cones or several semidefinite constraints. However, computable representations for such problems are not known. In our paper, we take the first step to handle such problems, i.e., one second-order cone constraint.

Another advantage in our paper is the use of second-order cone in the linear conic relaxation. In most literature, given a quadratic constraint, only the straightforward SDP relaxation is used. For example, a second-order cone constraint is relaxed to

$$
\left[\begin{array}{ccc}
0 & & \\
& -1 & \\
& & I
\end{array}\right] \bullet\left[\begin{array}{cc}
1 & y^{T} \\
y & Y
\end{array}\right] \leqslant 0, \quad\left[\begin{array}{cc}
1 & y^{T} \\
y & Y
\end{array}\right] \in \mathcal{S}_{+}^{n+2}
$$

where $\mathcal{S}_{+}^{n+2}$ is the set of positive semidefinite matrices of order $n+2$ and $M_{1} \bullet M_{2}$ being defined by $\operatorname{tr}\left(M_{1}^{T} M_{2}\right)$, the trace of $M_{1}^{T} M_{2}$. This formulation is only a relaxation. By adding an additional constraint $y \in \mathcal{S O C}(n)$, with $\mathcal{S O C}(n) \triangleq\left\{\left(x_{0}, x\right) \in\right.$ $\left.\mathbb{R} \times \mathbb{R}^{n} \mid\|x\| \leqslant x_{0}\right\}$, a tight representation can be obtained. Such an advantage has already been observed by several scholars recently (see $[2,5,8,13,14]$ ). In our paper, new results are based on such observation and the authors suggest that more attention be paid to the second-order cone constraint while constructing a linear conic relaxation.

In our derivation of computable representations, we adopt the concepts of copositive cone and cone of nonnegative quadratic functions which have been extensively used in recent studies. In [13], given a nonempty set $\mathcal{F} \subset \mathbb{R}^{n}$, the copositive cone over $\mathcal{F}$ is defined by

$$
\mathcal{H} \mathcal{D}_{\mathcal{F}} \triangleq\left\{M \in \mathcal{S}^{n} \mid x^{T} M x \geqslant 0, \forall x \in \mathcal{F}\right\}
$$

Its dual cone is

$$
\mathcal{H} \mathcal{D}_{\mathcal{F}}^{*}=\operatorname{cl} \text { cone }\left\{x x^{T} \in \mathcal{S}^{n} \mid x \in \mathcal{F}\right\},
$$


where "cl" means the closure and "cone" stands for the conic hull of a set (the smallest convex cone containing the given set). The cone of nonnegative quadratic functions over $\mathcal{F}$ is defined by

$$
\mathcal{D}_{\mathcal{F}} \triangleq\left\{M \in \mathcal{S}^{n+1} \mid M \bullet\left[\begin{array}{cc}
1 & x^{T} \\
x & x x^{T}
\end{array}\right] \geqslant 0, \forall x \in \mathcal{F}\right\} .
$$

Its dual cone has the formulation of

$$
\mathcal{D}_{\mathcal{F}}^{*}=\operatorname{cl} \text { cone }\left\{\left[\begin{array}{cc}
1 & x^{T} \\
x & x x^{T}
\end{array}\right] \in \mathcal{S}^{n+1} \mid x \in \mathcal{F}\right\} .
$$

The above four cones are all closed convex cones. They are related through the following set:

$$
\mathcal{H}_{\mathcal{F}}=\operatorname{cl}\left\{\left[\begin{array}{l}
t \\
x
\end{array}\right] \in \mathbb{R}^{n+1} \mid x / t \in \mathcal{F}, t>0\right\} .
$$

Sturm and Zhang [13] proved that

$$
\mathcal{D}_{\mathcal{F}}=\mathcal{H} \mathcal{D}_{\mathcal{H}_{\mathcal{F}}} \quad \text { and } \quad \mathcal{D}_{\mathcal{F}}^{*}=\mathcal{H D}_{\mathcal{H}_{\mathcal{F}}}^{*}
$$

They also showed that the QCQP problem has the same objective value as that of the following linear conic programming problem:

$$
\begin{gathered}
\inf \left[\begin{array}{cc}
c_{0} & b_{0}^{T} \\
b_{0} & A_{0}
\end{array}\right] \bullet Y \\
\text { s.t. } \quad Y_{11}=1 \\
Y \in \mathcal{D}_{\mathcal{F}}^{*}
\end{gathered}
$$

and that of its dual

$$
\begin{array}{ll}
\sup & \sigma \\
\text { s.t. } & {\left[\begin{array}{ll}
c_{0} & b_{0}^{T} \\
b_{0} & A_{0}
\end{array}\right]-\left[\begin{array}{cc}
\sigma & 0 \\
0 & 0
\end{array}\right] \in \mathcal{D}_{\mathcal{F}} \quad(\mathrm{LCoD})} \\
\sigma \in \mathbb{R} &
\end{array}
$$

Burer's copositive representation [1] worked on formulating the set $\mathcal{D}_{\mathcal{F}}^{*} \cap\left\{Y \mid Y_{11}=1\right\}$ with $\mathcal{F}=\left\{x \in \mathbb{R}^{n} \mid A x=b, x \in\{0,1\}^{n}\right\}$ under a key assumption of

$$
x \in\left\{y \in \mathbb{R}^{n} \mid A y=b, y \geqslant 0\right\} \quad \Longrightarrow \quad x \in\left\{y \in \mathbb{R}^{n} \mid 0 \leqslant y \leqslant 1\right\} .
$$

Burer [3] and Eichfelder and Povh [5] further extended the results to the case that $\mathcal{F}=\{x \mid A x=b, x \in \mathcal{K}\}$ with $\mathcal{K}$ being a closed convex cone. Their results can be used to construct the corresponding $\mathcal{D}_{\mathcal{F}}^{*}$. Based on [3] and [5], Burer and Dong [4] used the cone of nonnegative quadratic functions over the Cartesian product of several second-order cone constraints to represent some QCQP problems, which has been mentioned before. 
In the rest of the paper, some commonly used notation and properties of the cone of nonnegative quadratic functions are given in Sect. 2. An exact computable representation of the QCQP problem with one second-order cone constraint and two special linear constraints is provided in Sect. 3. Some concluding remarks follow in Sect. 4.

\section{Notations and Properties}

Given a nonempty set $\mathcal{F} \subseteq \mathbb{R}^{n}$, the cones $\mathcal{D}_{\mathcal{F}}, \mathcal{D}_{\mathcal{F}}^{*}, \mathcal{H} \mathcal{D}_{\mathcal{F}}, \mathcal{H} \mathcal{D}_{\mathcal{F}}^{*}$, and the set $\mathcal{H}_{\mathcal{F}}$ are respectively defined by (2)-(6). In this section, we first study the properties of these cones and then provide some useful tools for the proofs in Sect. 3.

\subsection{Properties of $\mathcal{D}_{\mathcal{F}}, \mathcal{D}_{\mathcal{F}}^{*}, \mathcal{H} \mathcal{D}_{\mathcal{F}}$ and $\mathcal{H} \mathcal{D}_{\mathcal{F}}^{*}$}

From [13], we have the next property.

Lemma 1 [13] Given a nonempty set $\mathcal{F} \subseteq \mathbb{R}^{n}$, we have the following facts: (i) $\mathcal{H}_{\mathcal{F}}$ is a closed cone; $($ ii $) \mathcal{D}_{\mathcal{F}}=\mathcal{H D}_{\mathcal{H}_{\mathcal{F}}} ;($ iii $) \mathcal{D}_{\mathcal{F}}^{*}=\mathcal{H D}_{\mathcal{H}_{\mathcal{F}}}^{*} ;$ (iv) $\mathcal{D}_{\mathcal{F}}$ and $\mathcal{D}_{\mathcal{F}}^{*}$ are dual to each other.

The closure operator in the definition of $\mathcal{H} \mathcal{D}_{\mathcal{F}}^{*}$ and $\mathcal{D}_{\mathcal{F}}^{*}$ is not desirable since it may be difficult to handle in an optimization problem. In some cases, the closeness requirement is automatically fulfilled without applying the closure operator. The next two lemmas provide necessary and sufficient conditions to omit the closure operator from the definition of $\mathcal{H} \mathcal{D}_{\mathcal{F}}^{*}$ and $\mathcal{D}_{\mathcal{F}}^{*}$ respectively.

Lemma 2 Given a nonempty set $\mathcal{F} \subseteq \mathbb{R}^{n}, \mathcal{H} \mathcal{D}_{\mathcal{F}}^{*}=\operatorname{cone}\left\{x x^{T} \in \mathcal{S}^{n} \mid x \in \operatorname{cl} \mathcal{F}\right\}$ if and only if $\operatorname{cl}\left\{t x \in \mathbb{R}^{n} \mid x \in \mathcal{F}, t \geqslant 0\right\}=\left\{t x \in \mathbb{R}^{n} \mid x \in \operatorname{cl} \mathcal{F}, t \geqslant 0\right\}$.

Proof It is clear that cone $\left\{x x^{T} \in \mathcal{S}^{n} \mid x \in \operatorname{cl} \mathcal{F}\right\} \subseteq \mathcal{H} \mathcal{D}_{\mathcal{F}}^{*}$ and $\left\{t x \in \mathbb{R}^{n} \mid x \in \operatorname{cl} \mathcal{F}\right.$, $t \geqslant 0\} \subseteq \operatorname{cl}\left\{t x \in \mathbb{R}^{n} \mid x \in \mathcal{F}, t \geqslant 0\right\}$.

["Only if" part] If $\mathcal{H} \mathcal{D}_{\mathcal{F}}^{*}=\operatorname{cone}\left\{x x^{T} \in \mathcal{S}^{n} \mid x \in \operatorname{cl} \mathcal{F}\right\}$, then, for any $y \in \operatorname{cl}\{t x \in$ $\left.\mathbb{R}^{n} \mid x \in \mathcal{F}, t \geqslant 0\right\}$, we have $y=\lim _{i \rightarrow+\infty} x^{i}$ where $x^{i} \in\left\{t x \in \mathbb{R}^{n} \mid x \in \mathcal{F}, t \geqslant 0\right\}$. Define $Y=y y^{T}$ and $X^{i}=x^{i}\left(x^{i}\right)^{T}$. Then $Y=\lim _{i \rightarrow+\infty} X^{i} \in \mathcal{H} \mathcal{D}_{\mathcal{F}}^{*}$. From our assumption, $Y \in \operatorname{cone}\left\{x x^{T} \in \mathcal{S}^{n} \mid x \in \operatorname{cl} \mathcal{F}\right\}$ and the rank of $Y$ is only 1 . Therefore, $Y=\lambda \bar{x} \bar{x}^{T}$ for some $\lambda \geqslant 0$ and $\bar{x} \in \operatorname{cl} \mathcal{F}$. This means that $y=\lambda^{\frac{1}{2}} \bar{x} \in\left\{t x \in \mathbb{R}^{n} \mid x \in \operatorname{cl} \mathcal{F}, t \geqslant 0\right\}$. Hence $\operatorname{cl}\left\{t x \in \mathbb{R}^{n} \mid x \in \mathcal{F}, t \geqslant 0\right\}=\left\{t x \in \mathbb{R}^{n} \mid x \in \operatorname{cl} \mathcal{F}, t \geqslant 0\right\}$.

["If" part] If $\operatorname{cl}\left\{t x \in \mathbb{R}^{n} \mid x \in \mathcal{F}, t \geqslant 0\right\}=\left\{t x \in \mathbb{R}^{n} \mid x \in \mathrm{cl} \mathcal{F}, t \geqslant 0\right\}$, then, for any $Y \in \mathcal{H D}_{\mathcal{F}}^{*}$, we have $Y=\lim _{i \rightarrow+\infty} Y^{i}$ where $Y^{i} \in \operatorname{cone}\left\{x x^{T} \in \mathcal{S}^{n} \mid x \in \mathcal{F}\right\}$ for all $i$. Notice that each $Y^{i}$ can be decomposed as $Y^{i}=\sum_{j=1}^{r_{i}}\left(\lambda_{j}^{i} x^{i j}\right)\left(\lambda_{j}^{i} x^{i j}\right)^{T}$ with $r_{i} \leqslant$ $\frac{n(n+1)}{2}, \lambda_{j}^{i} \geqslant 0$ and $x^{i j} \in \mathcal{F}$, for all $i, j$. Let $X^{i} \in \mathbb{R}^{n \times \frac{n(n+1)}{2}}$ be defined such that the first $r_{i}$ columns of $X^{i}$ are formed by $\left(\lambda_{j}^{i} x^{i j}\right), j=1, \cdots, r_{i}$, and the rest of columns are all zeros. Since $Y=\lim _{i \rightarrow+\infty} Y^{i}$ and $Y^{i}=X^{i}\left(X^{i}\right)^{T}$, we have $\lim _{i \rightarrow+\infty}\left(X^{i} \bullet\right.$ $\left.X^{i}\right)=\lim _{i \rightarrow+\infty} \operatorname{tr}\left(Y^{i}\right)=\operatorname{tr}(Y)$. Therefore, $\left\{X^{i}\right\}$ is a bounded sequence in $\mathbb{R}^{n \times \frac{n(n+1)}{2}}$ 
and there exists $\bar{X}$ which is the limit of a subsequence of $\left\{X^{i}\right\}$. Hence $Y=\bar{X} \bar{X}^{T}$. Notice that each column of $\bar{X}$ is an element of $\operatorname{cl}\left\{t x \in \mathbb{R}^{n} \mid x \in \mathcal{F}, t \geqslant 0\right\}$. From $\operatorname{cl}\{t x \in$ $\left.\mathbb{R}^{n} \mid x \in \mathcal{F}, t \geqslant 0\right\}=\left\{t x \in \mathbb{R}^{n} \mid x \in \mathrm{cl} \mathcal{F}\right\}$, each nonzero column of $\bar{X}$ can be denoted as $\lambda_{j} x^{j}$ with $\lambda_{j} \geqslant 0$ and $x^{j} \in \operatorname{cl} \mathcal{F}$. Consequently, $Y=\bar{X} \bar{X}^{T} \in \operatorname{cone}\left\{x x^{T} \in \mathcal{S}^{n} \mid x \in \operatorname{cl} \mathcal{F}\right\}$ and $\mathcal{H} \mathcal{D}_{\mathcal{F}}^{*}=\operatorname{cone}\left\{x x^{T} \in \mathcal{S}^{n} \mid x \in \operatorname{cl} \mathcal{F}\right\}$.

Remark 1 Given a set $\mathcal{F} \subseteq \mathbb{R}^{n}$, noticing that $\mathcal{H}_{\mathcal{F}}$ is a closed cone, hence we have $\mathcal{D}_{\mathcal{F}}^{*}=\mathcal{H} \mathcal{D}_{\mathcal{H}_{\mathcal{F}}}^{*}=\operatorname{cone}\left\{y y^{T} \in \mathcal{S}^{n+1} \mid y \in \mathcal{H}_{\mathcal{F}}\right\}=\operatorname{conv}\left\{y y^{T} \in \mathcal{S}^{n+1} \mid y \in \mathcal{H}_{\mathcal{F}}\right\}=$ $\left\{\sum_{i} y^{i}\left(y^{i}\right)^{T} \in \mathcal{S}^{n+1} \mid y^{i} \in \mathcal{H}_{\mathcal{F}}\right\}$. Therefore, showing $M \in \mathcal{D}_{\mathcal{F}}^{*}$ is equivalent to show$\operatorname{ing} M=\sum_{i} y^{i}\left(y^{i}\right)^{T}$ for some $y_{i} \in \mathcal{H}_{\mathcal{F}}$.

Remark 2 It was noticed in [6] that Lemma 1 of [13] does not always hold. Here we provide a necessary and sufficient condition for that Lemma. One may also check that Lemma 4 and Corollary 5 of [6] can be derived from our Lemma 2.

Lemma 3 Given a nonempty set $\mathcal{F} \subseteq \mathbb{R}^{n}, \mathcal{D}_{\mathcal{F}}^{*}=\operatorname{cones}\left\{\left[\begin{array}{cc}1 & x^{T} \\ x & x x^{T}\end{array}\right] \in \mathcal{S}^{n} \mid x \in \operatorname{cl} \mathcal{F}\right\}$ if and only if $\mathcal{F}$ is a bounded set.

Proof Since $\mathcal{D}_{\mathcal{F}}^{*}=\mathcal{H D}_{\mathcal{H}_{\mathcal{F}}}^{*}$ and $\mathcal{H}_{\mathcal{F}}=\operatorname{cl}\left\{t\left[\begin{array}{l}1 \\ x\end{array}\right] \mid x \in \mathcal{F}, t \geqslant 0\right\}$, we only need to prove that $\mathcal{H}_{\mathcal{F}}=\left\{\left[\begin{array}{l}t \\ x\end{array}\right] \in \mathbb{R}^{n+1} \mid x / t \in \operatorname{cl} \mathcal{F}, t>0\right\} \cup\{0\}$ if and only if $\mathcal{F}$ is bounded. Obviously, $\left\{\left[\begin{array}{l}t \\ x\end{array}\right] \in \mathbb{R}^{n+1} \mid x / t \in \operatorname{cl} \mathcal{F}, t>0\right\} \cup\{0\} \subseteq \mathcal{H}_{\mathcal{F}}$.

["If" part] When $\mathcal{F}$ is bounded, for any $y=\left[\begin{array}{l}t \\ x\end{array}\right] \in \mathcal{H}_{\mathcal{F}}$, we have $y=\lim _{i \rightarrow+\infty} y^{i}$ where $y^{i}=\left[\begin{array}{c}t^{i} \\ x^{i}\end{array}\right]$ with $t^{i}>0$ and $\frac{x^{i}}{t^{i}} \in \mathcal{F}$. (i) If $t=0$, then $\lim _{i \rightarrow+\infty} t^{i}=0$. Since $\mathcal{F}$ is bounded, the sequence $\left\{\frac{x^{i}}{t^{i}}\right\}$ is bounded. Therefore, $x=\lim _{i \rightarrow+\infty} t^{i} \frac{x^{i}}{t^{i}}=0$, i.e., $y=0$. (ii) If $t>0$, then, since $\left\{\frac{x^{i}}{t^{i}}\right\}$ is bounded, there exists a $z \in \operatorname{cl} \mathcal{F}$ being the limit of a subsequence of $\left\{\frac{x^{i}}{t^{i}}\right\}$. Hence $x=\lim _{i \rightarrow+\infty} t^{i} \frac{x^{i}}{t^{i}}=t z$, i.e., $y \in\left\{\left[\begin{array}{l}t \\ x\end{array}\right] \in \mathbb{R}^{n+1} \mid x / t \in\right.$ $\operatorname{cl} \mathcal{F}, t>0\}$. Therefore, $\mathcal{H}_{\mathcal{F}}=\left\{\left[\begin{array}{l}t \\ x\end{array}\right] \in \mathbb{R}^{n+1} \mid x / t \in \operatorname{cl} \mathcal{F}, t>0\right\} \cup\{0\}$.

["Only if" part] If $\mathcal{F}$ is unbounded, then there exists a sequence $\left\{z^{i}\right\}$ in $\mathcal{F}$ such that $\lim _{i \rightarrow+\infty}\left\|z^{i}\right\|=+\infty$. Without loss of generality, we may assume that none of these vectors is zero. Since the surface of the unit ball is closed and bounded, there exists $\bar{z}$ such that a subsequence of $\left\{\frac{z^{i}}{\left\|z^{i}\right\|}\right\}$ converges to $\bar{z}$. We can replace $\left\{z^{i}\right\}$ by such subsequence, i.e., we can assume that $\bar{z}=\lim _{i \rightarrow+\infty} \frac{z^{i}}{\left\|z^{i}\right\|} \neq 0$. Now define $y^{i}=$ $\left[\begin{array}{c}t^{i} \\ x^{i}\end{array}\right]=\left[\begin{array}{c}1 /\left\|z^{i}\right\| \\ z^{i} /\left\|z^{i}\right\|\end{array}\right]$. We have $\lim _{i \rightarrow+\infty} y^{i}=\left[\begin{array}{l}0 \\ \bar{z}\end{array}\right] \in \mathcal{H}_{\mathcal{F}}$. However, $\left[\begin{array}{l}0 \\ \bar{z}\end{array}\right] \notin\left\{\left[\begin{array}{l}t \\ x\end{array}\right] \in \mathbb{R}^{n+1} \mid x / t \in\right.$ $\operatorname{cl} \mathcal{F}, t>0\} \cup\{0\}$. Therefore, $\mathcal{H}_{\mathcal{F}} \neq\left\{\left[\begin{array}{l}t \\ x\end{array}\right] \in \mathbb{R}^{n+1} \mid x / t \in \operatorname{cl} \mathcal{F}, t>0\right\} \cup\{0\}$.

Together with Lemma 2, we have $\mathcal{D}_{\mathcal{F}}^{*}=\operatorname{cone}\left\{\left[\begin{array}{cc}1 & x^{T} \\ x & x x^{T}\end{array}\right] \in \mathcal{S}^{n} \mid x \in \operatorname{cl} \mathcal{F}\right\}$ if and only if $\mathcal{H}_{\mathcal{F}}=\left\{\left[\begin{array}{l}t \\ x\end{array}\right] \in \mathbb{R}^{n+1} \mid x / t \in \operatorname{cl} \mathcal{F}, t>0\right\} \cup\{0\}$, which is equivalent to saying that $\mathcal{F}$ is bounded.

As we can see, the cone of nonnegative quadratic functions and its dual cone posses the following monotonic properties: 
Lemma 4 If $\mathcal{F}_{1} \subseteq \mathcal{F}_{2} \subseteq \mathbb{R}^{n}$, then $\mathcal{D}_{\mathcal{F}_{1}}^{*} \subseteq \mathcal{D}_{\mathcal{F}_{2}}^{*}$ and $\mathcal{D}_{\mathcal{F}_{1}} \supseteq \mathcal{D}_{\mathcal{F}_{2}}$. Moreover, for any given $\mathcal{F} \subseteq \mathbb{R}^{n}, \mathcal{D}_{\mathcal{F}}^{*} \subseteq \mathcal{S}_{+}^{n+1} \subseteq \mathcal{D}_{\mathcal{F}}$.

Proof The proof follows directly from the definitions (2)-(5).

Given a set $K$, we use $K^{*}$ to denote its dual set, which is a closed convex cone. The next lemma will be needed in Lemma 6 and later proofs in Sect. 3.

Lemma 5 (Corollary 16.4.2 in [12]) If $K_{1}, \cdots, K_{s}$ are nonempty closed convex cones in $\mathbb{R}^{n}$, then

$$
\left(\bigcap_{i=1}^{s} K_{i}\right)^{*}=\operatorname{cl}\left(\sum_{i=1}^{s} K_{i}^{*}\right) .
$$

If there exists a common point of the relative interior of each $K_{i}, i=1, \cdots, s$, then

$$
\left(\bigcap_{i=1}^{s} K_{i}\right)^{*}=\left(\sum_{i=1}^{s} K_{i}^{*}\right) .
$$

Together with Lemma 4 and Lemma 5, we have the next result.

Lemma 6 If $\mathcal{F}=\bigcup_{i=1}^{k} \mathcal{F}_{i} \subseteq \mathbb{R}^{n}$ and each $\mathcal{F}_{i}$ is nonempty, then $\mathcal{D}_{\mathcal{F}}=\bigcap_{i=1}^{k} \mathcal{D}_{\mathcal{F}_{i}}$ and $\mathcal{D}_{\mathcal{F}}^{*}=\sum_{i=1}^{k} \mathcal{D}_{\mathcal{F}_{i}}^{*}$.

Proof From Lemma 4, we have $\mathcal{D}_{\mathcal{F}} \subseteq \mathcal{D}_{\mathcal{F}_{i}}$ for $i=1, \cdots, k$. Consequently, $\mathcal{D}_{\mathcal{F}} \subseteq$ $\bigcap_{i=1}^{k} \mathcal{D}_{\mathcal{F}_{i}}$. Now if $M \in \bigcap_{i=1}^{k} \mathcal{D}_{\mathcal{F}_{i}}$, then, from $M \in \mathcal{D}_{\mathcal{F}_{i}}$, we know $M \bullet\left[\begin{array}{cc}1 & x^{T} \\ x & x x^{T}\end{array}\right] \geqslant 0$ for each $x \in \mathcal{F}_{i}$, which means $M \bullet\left[\begin{array}{cc}1 & x^{T} \\ x & x x^{T}\end{array}\right] \geqslant 0$ for all $x \in \bigcup_{i=1}^{k} \mathcal{F}_{i}=\mathcal{F}$. Therefore, $M \in \mathcal{D}_{\mathcal{F}}$ and $\mathcal{D}_{\mathcal{F}} \supseteq \bigcap_{i=1}^{k} \mathcal{D}_{\mathcal{F}_{i}}$. Consequently, $\mathcal{D}_{\mathcal{F}}=\bigcap_{i=1}^{k} \mathcal{D}_{\mathcal{F}_{i}}$.

Notice that $\mathcal{D}_{\mathcal{F}_{i}}$ is a closed convex cone and $\mathcal{D}_{\mathcal{F}_{i}} \supseteq \mathcal{S}_{+}^{n+1}, i=1, \cdots, k$. From Lemma 5, we have $\sum_{i=1}^{k} \mathcal{D}_{\mathcal{F}_{i}}^{*}=\left(\bigcap_{i=1}^{k} \mathcal{D}_{\mathcal{F}_{i}}\right)^{*}=\left(\mathcal{D}_{\mathcal{F}}\right)^{*}=\mathcal{D}_{\mathcal{F}}^{*}$.

When $\mathcal{F}=\mathcal{F}_{1} \times \mathbb{R}^{m}$ for some positive integer $m, \mathcal{D}_{\mathcal{F}}^{*}$ can be expressed by $\mathcal{D}_{\mathcal{F}_{1}}^{*}$ and one additional semidefinite constraint as in the next lemma.

Lemma 7 Given a nonempty set $\mathcal{F}_{1} \subseteq \mathbb{R}^{n}$ and let $\mathcal{F}=\mathcal{F}_{1} \times \mathbb{R}^{m}$, then

$$
\mathcal{D}_{\mathcal{F}}^{*}=\left\{\left[\begin{array}{cc}
A_{1} & A_{2}^{T} \\
A_{2} & A_{3}
\end{array}\right] \in \mathcal{S}_{+}^{1+n+m} \mid A_{1} \in \mathcal{D}_{\mathcal{F}_{1}}^{*}\right\} .
$$

Proof Let

$$
\mathcal{K}=\left\{\left[\begin{array}{cc}
A_{1} & A_{2}^{T} \\
A_{2} & A_{3}
\end{array}\right] \in \mathcal{S}_{+}^{1+n+m} \mid A_{1} \in \mathcal{D}_{\mathcal{F}_{1}}^{*}\right\}
$$


Since $\mathcal{H}_{\mathcal{F}}=\mathcal{H}_{\mathcal{F}_{1}} \times \mathbb{R}^{m}$ and

$$
\mathcal{D}_{\mathcal{F}}^{*}=\left\{\sum_{i}\left[\begin{array}{l}
u^{i} \\
v^{i}
\end{array}\right]\left[\begin{array}{l}
u^{i} \\
v^{i}
\end{array}\right]^{T} \in \mathcal{S}_{+}^{1+n+m} \mid\left[\begin{array}{l}
u^{i} \\
v^{i}
\end{array}\right] \in \mathcal{H}_{\mathcal{F}}=\mathcal{H}_{\mathcal{F}_{1}} \times \mathbb{R}^{m}\right\},
$$

we have $Y=\sum_{i}\left[\begin{array}{l}u^{i} \\ v^{i}\end{array}\right]\left[\begin{array}{l}u^{i} \\ v^{i}\end{array}\right]^{T}=\left[\begin{array}{cc}Y_{1} & Y_{2}^{T} \\ Y_{2} & Y_{3}\end{array}\right] \in \mathcal{S}_{+}^{1+n+m}$, for any $Y \in \mathcal{D}_{\mathcal{F}}^{*}$, and $Y_{1}=$ $\sum_{i} u^{i}\left(u^{i}\right)^{T} \in \mathcal{D}_{\mathcal{F}_{1}}^{*}$. Therefore, $Y \in \mathcal{K}$ and $\mathcal{D}_{\mathcal{F}}^{*} \subseteq \mathcal{K}$.

Moreover, if $Y=\left[\begin{array}{cc}Y_{1} & Y_{2}^{T} \\ Y_{2} & Y_{3}\end{array}\right] \in \mathcal{K}$, then $Y \in \mathcal{S}_{+}^{1+n+m}$ and $Y_{1} \in \mathcal{D}_{\mathcal{F}_{1}}^{*}$. We can find decompositions $Y_{1}=P P^{T}=B B^{T}$, where $P \in \mathbb{R}^{(1+n) \times k}$ for some $k>0$ with each column of $P$ lying in $\mathcal{H}_{\mathcal{F}_{1}}$ and $B \in \mathbb{R}^{(1+n) \times r}$ with $r=\operatorname{rank}\left(Y_{1}\right)$. Furthermore, we have $r \leqslant k$ and $P=B Q$ for some $Q \in \mathbb{R}^{r \times k}$ being of full row rank. Since $Y$ is positive semidefinite, there exists $R \in \mathbb{R}^{r \times m}$ such that $Y_{2}^{T}=B R$. Hence

$$
Y=\left[\begin{array}{cc}
B B^{T} & B R \\
R^{T} B^{T} & Y_{3}
\end{array}\right]=\left[\begin{array}{cc}
B B^{T} & B R \\
R^{T} B^{T} & R^{T} R
\end{array}\right]+\left[\begin{array}{cc}
0 & 0 \\
0 & Y_{3}-R^{T} R
\end{array}\right] .
$$

Notice that $Y \in \mathcal{S}_{+}^{1+n+m}$ if and only if $Y_{3}-R^{T} R \in \mathcal{S}_{+}^{m}$. (Otherwise, $\bar{z}=$ $\left[\begin{array}{c}-B\left(B^{T} B\right)^{-1} R \bar{v} \\ \bar{v}\end{array}\right] \in \mathbb{R}^{(1+n+m)}$ with $\bar{v}^{T}\left(Y_{3}-R^{T} R\right) \bar{v}<0$ disproves the positive semidefiniteness of $Y$ due to the fact that $\bar{z}^{T} Y \bar{z}=\bar{v}^{T}\left(Y_{3}-R^{T} R\right) \bar{v}<0$.) Clearly, $\left[\begin{array}{cc}0 & 0 \\ 0 & Y_{3}-R^{T} R\end{array}\right] \in \mathcal{D}_{\mathcal{F}}^{*}$. We now prove that $\left[\begin{array}{ccc}B B^{T} & B R \\ R^{T} B^{T} & R^{T} R\end{array}\right] \in \mathcal{D}_{\mathcal{F}}^{*}$. Since $B B^{T}=P P^{T}=$ $B Q Q^{T} B^{T}$, we have

$$
\begin{aligned}
Q Q^{T} & =\left(B^{T} B\right)^{-1} B^{T}\left(B Q Q^{T} B^{T}\right) B\left(B^{T} B\right)^{-1} \\
& =\left(B^{T} B\right)^{-1} B^{T} B B^{T} B\left(B^{T} B\right)^{-1}=I_{r} .
\end{aligned}
$$

Let $U=R^{T} Q$, then

$$
\left[\begin{array}{l}
P \\
U
\end{array}\right]\left[\begin{array}{l}
P \\
U
\end{array}\right]^{T}=\left[\begin{array}{ll}
P P^{T} & P U^{T} \\
U P^{T} & U U^{T}
\end{array}\right]=\left[\begin{array}{cc}
B B^{T} & B R \\
R^{T} B^{T} & R^{T} R
\end{array}\right]
$$

Notice that each column of $\left[\begin{array}{l}P \\ U\end{array}\right]$ is in $\mathcal{H}_{\mathcal{F}}$. Hence $\left[\begin{array}{cc}B B^{T} & B R \\ R^{T} B^{T} & R^{T} R\end{array}\right] \in \mathcal{D}_{\mathcal{F}}^{*}$. This leads to $Y \in \mathcal{D}_{\mathcal{F}}^{*}$ and $\mathcal{K} \subseteq \mathcal{D}_{\mathcal{F}}^{*}$. Together with $\mathcal{D}_{\mathcal{F}}^{*} \subseteq \mathcal{K}$, we have $\mathcal{D}_{\mathcal{F}}^{*}=\mathcal{K}$.

Burer [3] proved that when $\mathcal{F}=\left\{x \in \mathcal{K} \subseteq \mathbb{R}^{n} \mid A x=b\right\}$ with $\mathcal{K}$ being a closed convex cone, then

$\mathcal{D}_{\mathcal{F}}^{*} \cap\left\{Y \in \mathcal{S}^{n+1} \mid Y_{11}=1\right\}=\left\{Y=\left[\begin{array}{cc}1 & x^{T} \\ x & X\end{array}\right] \in \mathcal{D}_{\mathcal{K}}^{*} \subseteq \mathcal{S}^{n+1} \mid \begin{array}{c}A x=b \\ \operatorname{diag}\left(A X A^{T}\right)=b \circ b\end{array}\right\}$

where $\operatorname{diag}(M)$ is a vector with $[\operatorname{diag}(M)]_{i}=M_{i i}, i=1, \cdots, n$, and $b \circ b$ is a vector with $[b \circ b]_{i}=b_{i}^{2}, i=1, \cdots, m$. Here we give a more general result on $\mathcal{D}_{\mathcal{F}}^{*}$ and the proof is similar to that of Burer [3]. 
Lemma 8 Given $\mathcal{F}_{0} \subseteq \mathbb{R}^{n}, A \in \mathbb{R}^{m \times n}$ and $b \in \mathbb{R}^{m}$, if $\mathcal{F}=\left\{x \in \mathcal{F}_{0} \mid A x=b\right\}$ is a nonempty set and $\mathcal{H}_{\mathcal{F}}=\mathcal{H}_{\mathcal{F}_{0}} \cap\left\{\left[\begin{array}{l}t \\ x\end{array}\right] \in \mathbb{R}^{n+1} \mid A x=t b\right\}$, then

$$
\mathcal{D}_{\mathcal{F}}^{*}=\left\{Y=\left[\begin{array}{cc}
\chi & x^{T} \\
x & X
\end{array}\right] \in \mathcal{D}_{\mathcal{F}_{0}}^{*} \subseteq \mathcal{S}^{n+1} \mid \begin{array}{c}
A x=\chi b \\
\operatorname{diag}\left(A X A^{T}\right)=\chi(b \circ b)
\end{array}\right\} .
$$

Proof Define

$$
\mathcal{G} \triangleq\left\{Y=\left[\begin{array}{cc}
\chi & x^{T} \\
x & X
\end{array}\right] \in \mathcal{D}_{\mathcal{F}_{0}}^{*} \subseteq \mathcal{S}^{n+1} \mid \begin{array}{c}
A x=\chi b \\
\operatorname{diag}\left(A X A^{T}\right)=\chi(b \circ b)
\end{array}\right\} .
$$

Since $\left[\begin{array}{cc}1 & x^{T} \\ x & x x^{T}\end{array}\right] \in \mathcal{G}$ for any $x \in \mathcal{F}$ and $\mathcal{G}$ is a closed convex cone, we have $\mathcal{D}_{\mathcal{F}}^{*} \subseteq \mathcal{G}$.

For the reverse direction, it is sufficient to show that every $Y \in \mathcal{G}$ can be represented as

$$
Y=\sum_{i} y^{i}\left(y^{i}\right)^{T}
$$

with $y^{i} \in \mathcal{H}_{\mathcal{F}}$. As we can see that

$$
\mathcal{D}_{\mathcal{F}_{0}}^{*}=\operatorname{cone}\left\{y y^{T} \in \mathcal{S}^{n+1} \mid y \in \mathcal{H}_{\mathcal{F}_{0}}\right\}=\left\{\sum_{i} y^{i}\left(y^{i}\right)^{T} \in \mathcal{S}^{n+1} \mid y^{i} \in \mathcal{H}_{\mathcal{F}_{0}}\right\} .
$$

For any $Y \in \mathcal{G}$, we have

$$
Y=\sum_{i} y^{i}\left(y^{i}\right)^{T}=\sum_{i}\left[\begin{array}{l}
\xi^{i} \\
z^{i}
\end{array}\right]\left[\begin{array}{l}
\xi^{i} \\
z^{i}
\end{array}\right]^{T}
$$

with $\xi^{i} \geqslant 0$ and $\left[\begin{array}{c}\xi^{i} \\ z^{i}\end{array}\right] \in \mathcal{H}_{\mathcal{F}_{0}}$. We claim that: (i) if $\xi^{i}=0$, then $z^{i}$ satisfies that $A z^{i}=0$ and $\left[\begin{array}{c}0 \\ z^{i}\end{array}\right] \in \mathcal{H}_{\mathcal{F}}$; (ii) if $\xi^{i}>0$, then $x^{i}=z^{i} / \xi^{i}$ satisfies that $A x^{i}=b$ and $\left[\begin{array}{c}\xi^{i} \\ z^{i}\end{array}\right] \in \mathcal{H}_{\mathcal{F}}$.

Since $Y \in \mathcal{G}$, we have

$$
\left(\sum_{i}\left(\xi^{i}\right)^{2}\right) b=\sum_{i} \xi^{i} A z^{i}
$$

and

$$
\left(\sum_{i}\left(\xi^{i}\right)^{2}\right) b \circ b=\sum_{i} \operatorname{diag}\left(A\left(z^{i}\left(z^{i}\right)^{T}\right) A^{T}\right)=\sum_{i}\left(A z^{i}\right) \circ\left(A z^{i}\right) .
$$

Consequently,

$$
\left(\sum_{i} \xi^{i} A z^{i}\right) \circ\left(\sum_{i} \xi^{i} A z^{i}\right)=\left(\sum_{i}\left(\xi^{i}\right)^{2}\right) \sum_{i}\left(A z^{i}\right) \circ\left(A z^{i}\right) .
$$

By the Cauchy-Schwarz inequality, the equality sign holds if and only if there exists a $\delta \in \mathbb{R}^{m}$ such that $\xi^{i} \delta=A z^{i}$ for all $i$. 
When $\xi^{i}=0$, we have $A z^{i}=0$. From the assumption on $\mathcal{H}_{\mathcal{F}}$, we know that $\left[\begin{array}{c}0 \\ z^{i}\end{array}\right] \in$ $\mathcal{H}_{\mathcal{F}}$ and Claim (i) holds.

When $\xi^{i}>0$, we only need to prove that $\delta=b$. Notice that

$$
\left(\sum_{j}\left(\xi^{j}\right)^{2}\right) b=\sum_{j} \xi^{j} A z^{j}=\left(\sum_{j}\left(\xi^{j}\right)^{2}\right) \delta .
$$

Since $\xi^{i}>0$, the above equation leads to $\delta=b$. This proves Claim (ii).

From Claims (i) and (ii), we have $Y \in \mathcal{D}_{\mathcal{F}}^{*}$ and $\mathcal{G} \subseteq \mathcal{D}_{\mathcal{F}}^{*}$. Together with $\mathcal{D}_{\mathcal{F}}^{*} \subseteq \mathcal{G}$, we have $\mathcal{D}_{\mathcal{F}}^{*}=\mathcal{G}$.

Remark 3 When $\mathcal{F}_{0}$ is a closed convex cone or a closed bounded set, the assumption on $\mathcal{H}_{\mathcal{F}}$ always holds. Consequently, the study on the representation of $\mathcal{D}_{\mathcal{F}}^{*}$ can be simplified to the one of $\mathcal{D}_{\mathcal{F}_{0}}^{*}$.

Remark 4 According to Lemmas 2, 6, 7 and 8, when deriving computable representations, (i) showing $M \in \mathcal{D}_{\mathcal{F}}^{*}$ is equivalent to showing $M=\sum_{i} y^{i}\left(y^{i}\right)^{T}$ for some $y_{i} \in \mathcal{H}_{\mathcal{F}}$; (2) if $\mathcal{F}$ is the union of several sets, we could treat them separately; (3) we could focus on the set without linear equality constraints (under certain conditions) and free variables. These properties will simplify the proof of the computable representation.

\subsection{Some Useful Results}

In this subsection, we introduce some results used in the proofs in Sect. 3.

Firstly, three observations can be made here: (i) Given a nonempty set $\mathcal{F} \subseteq \mathbb{R}^{n}$ and a closed convex cone $\mathcal{K} \subseteq \mathcal{S}_{+}^{n+1}$, if $\mathcal{D}_{\mathcal{F}}^{*} \subseteq \mathcal{K}$, in order to prove $\mathcal{D}_{\mathcal{F}}^{*}=\mathcal{K}$, we only need to prove that $\mathcal{K}^{\prime} \triangleq \mathcal{K} \cap\left\{Y \in \mathcal{S}^{n+1} \mid \operatorname{tr}(Y) \leqslant 1\right\} \subset \mathcal{D}_{\mathcal{F}}^{*}$. (ii) Given $Y=Y^{1}+Y^{2}$ with $Y, Y^{1}, Y^{2} \neq 0, Y \in \mathcal{K}^{\prime}$ and $Y^{1}, Y^{2} \in \mathcal{K} \subseteq \mathcal{S}_{+}^{n+1}$, a convex combination of $Y$ can be obtained by reformulating $Y=\frac{\operatorname{tr}\left(Y^{1}\right)}{\operatorname{tr}(Y)} Z^{1}+\frac{\operatorname{tr}\left(Y^{2}\right)}{\operatorname{tr}(Y)} Z^{2}$ with $Z^{1}=\frac{\operatorname{tr}(Y)}{\operatorname{tr}\left(Y^{1}\right)} Y^{1} \in \mathcal{K}^{\prime}$ and $Z^{2}=\frac{\operatorname{tr}(Y)}{\operatorname{tr}\left(Y^{2}\right)} Y^{2} \in \mathcal{K}^{\prime}$. (iii) Since $\mathcal{K}^{\prime}$ is a bounded closed convex set, the task of proving $\mathcal{K}^{\prime} \subset \mathcal{D}_{\mathcal{F}}^{*}$ can be reduced to proving that every extreme point of $\mathcal{K}^{\prime}$ is contained in $\mathcal{D}_{\mathcal{F}}^{*}$.

The next lemma characterizes the property of the extreme points for an SDP feasible set.

Lemma 9 [11] Consider an SDP feasible set, for some integer $p>0$ and $A^{i j} \in \mathcal{S}^{n_{j}}$, $i=1, \cdots, m, j=1, \cdots, p$, let

$$
F \triangleq\left\{\left(X^{1}, \cdots, X^{p}\right) \in \mathcal{S}_{+}^{n_{1}} \times \cdots \times \mathcal{S}_{+}^{n_{p}} \mid \sum_{j=1}^{p} A^{i j} \bullet X^{j}=b_{i}, i=1, \cdots, m\right\} .
$$

If $\left(X^{1}, \cdots, X^{p}\right)$ is an extreme point of $F$ and $r_{j}=\operatorname{rank}\left(X^{j}\right)$, then $\sum_{j=1}^{p} r_{j}\left(r_{j}+1\right) \leqslant$ $2 m$. 
In order to investigate the second-order cone constraint through the above lemma, we need its equivalent SDP representation.

Lemma 10 [2] Given $z_{0} \in \mathbb{R}$ and $z \in \mathbb{R}^{n}$, let

$$
\operatorname{Arrow}\left(z_{0}, z\right) \triangleq\left[\begin{array}{cc}
z_{0} I_{n} & z \\
z^{T} & z_{0}
\end{array}\right]
$$

and $r=\operatorname{rank}\left(\operatorname{Arrow}\left(z_{0}, z\right)\right)$. Then $\|z\| \leqslant z_{0}$ if and only if $\operatorname{Arrow}\left(z_{0}, z\right) \in \mathcal{S}_{+}^{n+1}$. In addition, if $\|z\| \leqslant z_{0}$, then one of the following three cases holds: $(i)\left(z_{0}, z\right)=0$ and $r=0$; (ii) $\|z\|=z_{0}>0$ and $r=n$; (iii) $\|z\|<z_{0}$ and $r=n+1$.

The next result about rank-one decomposition will be used repeatedly in later proofs.

Lemma 11 Let $X \in \mathcal{S}_{+}^{n}$ be a nonzero matrix and $\operatorname{rank}(X)=r$. For any vector $a \in \mathbb{R}^{n}$, if $X a \neq 0$, then $X^{\prime}=X-\frac{X a a^{T} X}{a^{T} X a} \in \mathcal{S}_{+}^{n}$ and $\operatorname{rank}\left(X^{\prime}\right)=r-1$.

Proof Let $X=Y^{T} Y$. The first claim can be proved by noticing that $\left(u^{T} X u\right) \times$ $\left(a^{T} X a\right)=\|Y u\|^{2}\|Y a\|^{2} \geqslant\left((Y u)^{T}(Y a)\right)^{2}=\left(u^{T} X a\right)^{2}$, for any $u \in \mathbb{R}^{n}$.

Obviously, $\operatorname{rank}\left(X^{\prime}\right) \geqslant r-1$. The second claim can be proved by noticing that (i) any $u$ in the null space of $X$ is also in the null space of $X^{\prime}$; (ii) $a$ is in the null space of $X^{\prime}$ but not in the null space of $X$.

\section{QCQP with One Second-Order Cone Constraint}

In this section, we focus on the exact computable representation of the QCQP problem whose domain is defined by one second-order cone constraint and some special linear constraints.

Our first result deals the QCQP problem whose domain is specified by one secondorder cone constraint and one special linear constraint.

Theorem 1 Given a nonempty set $\mathcal{F}=\left\{(x, y) \in \mathbb{R}^{n_{1}} \times \mathbb{R}^{n_{2}} \mid\|x\| \leqslant a_{1}+a_{2}^{T} x+\right.$ $\left.a_{3}^{T} y, a_{1}+a_{2}^{T} x+a_{3}^{T} y \geqslant a_{4} \geqslant 0\right\}$ with $a_{1} \in \mathbb{R}, a_{2} \in \mathbb{R}^{n_{1}}, a_{3} \in \mathbb{R}^{n_{2}}$ and $a_{4} \geqslant 0$, let

$$
\begin{aligned}
& a^{T} \triangleq\left[\begin{array}{lll}
a_{1} & a_{2}^{T} & a_{3}^{T}
\end{array}\right], \\
& b \triangleq a-a_{4} e_{1}, \\
& e_{1} \triangleq(1,0, \cdots, 0)^{T} \in \mathbb{R}^{1+n_{1}+n_{2}}, \\
& C_{1} \triangleq\left[\begin{array}{lll}
0 & I_{n_{1}} & 0
\end{array}\right], \\
& C_{2} \triangleq\left[\begin{array}{ll}
a & C_{1}^{T}
\end{array}\right], \\
& C_{3} \triangleq a a^{T}-C_{1}^{T} C_{1} .
\end{aligned}
$$


Then we have

$\mathcal{D}_{\mathcal{F}}^{*}=\left\{U=\left[\begin{array}{ccc}\chi & x^{T} & y^{T} \\ x & X & W^{T} \\ y & W & Y\end{array}\right] \in \mathcal{S}_{+}^{1+n_{1}+n_{2}} \mid \begin{array}{c}\left\|C_{1} U e_{1}\right\| \leqslant a^{T} U e_{1}, U \\ \bullet\left(a a^{T}-C_{1}^{T} C_{1}\right) \geqslant 0, \\ b^{T} U e_{1} \geqslant 0, a^{T} U b \geqslant\left\|C_{1} U b\right\|\end{array}\right\}$,

$\mathcal{D}_{\mathcal{F}}=\mathrm{cl}\left\{M \in \mathcal{S}^{1+n_{1}+n_{2}} \mid \begin{array}{l}M-\lambda_{1} C_{3}-\lambda_{2}\left(e_{1} b^{T}+b e_{1}^{T}\right)-\left(e_{1} \psi_{1}^{T} C_{2}^{T}+C_{2} \psi_{1} e_{1}^{T}\right) \\ -\left(b \psi_{2}^{T} C_{2}^{T}+C_{2} \psi_{2} b^{T}\right) \in \mathcal{S}_{+}^{1+n_{1}+n_{2}}, \\ \lambda_{1}, \lambda_{2} \geqslant 0, \psi_{1}, \psi_{2} \in \mathcal{S O C}\left(n_{1}\right)\end{array}\right\}$.

Moreover, the corresponding problems of QCQP and LCoP defined in (1) and (8), respectively, have the same optimal value.

If there exists $(\bar{x}, \bar{y}) \in \mathbb{R}^{n_{1}} \times \mathbb{R}^{n_{2}}$ such that $\|\bar{x}\|<a_{1}+a_{2}^{T} \bar{x}+a_{3}^{T} \bar{y}$ and $a_{1}+a_{2}^{T} \bar{x}+$ $a_{3}^{T} \bar{y}>a_{4}$, then $\mathcal{D}_{\mathcal{F}}$ can be simplified as

$$
\mathcal{D}_{\mathcal{F}}=\left\{\begin{array}{l|l}
M \in \mathcal{S}^{1+n_{1}+n_{2}} & \begin{array}{l}
M-\lambda_{1} C_{3}-\lambda_{2}\left(e_{1} b^{T}+b e_{1}^{T}\right)-\left(e_{1} \psi_{1}^{T} C_{2}^{T}+C_{2} \psi_{1} e_{1}^{T}\right) \\
-\left(b \psi_{2}^{T} C_{2}^{T}+C_{2} \psi_{2} b^{T}\right) \in \mathcal{S}_{+}^{1+n_{1}+n_{2}}, \\
\lambda_{1}, \lambda_{2} \geqslant 0, \psi_{1}, \psi_{2} \in \mathcal{S O C}\left(n_{1}\right)
\end{array}
\end{array}\right\} .
$$

Moreover, the corresponding dual problem LCoD (as defined in (9))

$\sup \sigma$

$$
\begin{aligned}
\text { s.t. } & {\left[\begin{array}{ll}
c_{0} & b_{0}^{T} \\
b_{0} & A_{0}
\end{array}\right]-\left[\begin{array}{cc}
\sigma & 0 \\
0 & 0
\end{array}\right]-\lambda_{1} C_{3}-\lambda_{2}\left(e_{1} b^{T}+b e_{1}^{T}\right) } \\
& -\left(e_{1} \psi_{1}^{T} C_{2}^{T}+C_{2} \psi_{1} e_{1}^{T}\right)-\left(b \psi_{2}^{T} C_{2}^{T}+C_{2} \psi_{2} b^{T}\right) \in \mathcal{S}_{+}^{1+n_{1}+n_{2}}
\end{aligned}
$$

$\sigma \in \mathbb{R}, \lambda_{1}, \lambda_{2} \geqslant 0, \psi_{1}, \psi_{2} \in \mathcal{S O C}\left(n_{1}\right)$

attains the same optimal value as that of the original problem QCQP.

Proof Define

$$
\mathcal{K} \triangleq\left\{\begin{array}{l|cc}
U=\left[\begin{array}{ccc}
\chi & x^{T} & y^{T} \\
x & X & W^{T} \\
y & W & Y
\end{array}\right] \in \mathcal{S}_{+}^{1+n_{1}+n_{2}} & \begin{array}{l}
a^{T} U e_{1} \geqslant\left\|C_{1} U e_{1}\right\|, \\
U \bullet\left(a a^{T}-C_{1}^{T} C_{1}\right) \geqslant 0, \\
b^{T} U e_{1} \geqslant 0, a^{T} U b \geqslant\left\|C_{1} U b\right\|
\end{array}
\end{array}\right\} .
$$

It is clear that $\mathcal{D}_{\mathcal{F}}^{*} \subseteq \mathcal{K}$.

To prove $\mathcal{K} \subseteq \mathcal{D}_{\mathcal{F}}^{*}$, it is sufficient to show that all the extreme points of $\mathcal{K}^{\prime} \triangleq \mathcal{K} \cap$ $\left\{U \in \mathcal{S}^{1+n_{1}+n_{2}} \mid \operatorname{tr} U \leqslant 1\right\}$ belong to $\mathcal{D}_{\mathcal{F}}^{*}$. In other word, for each nonzero extreme point of $\mathcal{K}^{\prime}$, we need to find a rank-one decomposition with all elements falling in $\mathcal{H}_{\mathcal{F}}$.

We first prove that

$$
\begin{aligned}
& \left\{(t, x, y) \in \mathbb{R}_{+} \times \mathbb{R}^{n_{1}} \times \mathbb{R}^{n_{2}} \mid\|x\| \leqslant a_{1} t+a_{2}^{T} x+a_{3}^{T} y,\right. \\
& \left.a_{1} t+a_{2}^{T} x+a_{3}^{T} y \geqslant a_{4} t, t \geqslant 0\right\} \subseteq \mathcal{H}_{\mathcal{F}} .
\end{aligned}
$$


If $t>0$, then $\left[\begin{array}{l}x / t \\ y / t\end{array}\right] \in \mathcal{F}$ and hence $\left[\begin{array}{l}t \\ x \\ y\end{array}\right] \in \mathcal{H}_{\mathcal{F}}$. Otherwise, if $t=0$, since $\mathcal{F}$ is not empty, there exists $\left[\begin{array}{l}\bar{x} \\ \bar{y}\end{array}\right] \in \mathcal{F}$. One can verify that $\left[\begin{array}{l}0 \\ x \\ y\end{array}\right]+\frac{1}{k}\left[\begin{array}{l}1 \\ \bar{x} \\ \bar{y}\end{array}\right] \in \mathcal{H}_{\mathcal{F}}$. When $k$ goes to infinity, its limit $\left[\begin{array}{l}0 \\ x \\ y\end{array}\right] \in \mathcal{H}_{\mathcal{F}}$. Therefore, the above inclusion holds true.

Next, we let $U^{0}$ be a nonzero extreme point of $\mathcal{K}^{\prime}$ and consider the following five cases for a complete proof: (i) $\chi=0$; (ii) $\chi>0, a^{T} U e_{1}=\left\|C_{1} U e_{1}\right\|$ and $a^{T} U b=\left\|C_{1} U b\right\|$; (iii) $\chi>0, a^{T} U e_{1}>\left\|C_{1} U e_{1}\right\|$ and $a^{T} U b=\left\|C_{1} U b\right\|$; (iv) $\chi>0, a^{T} U e_{1}=\left\|C_{1} U e_{1}\right\|$ and $a^{T} U b>\left\|C_{1} U b\right\|$; (v) $\chi>0, a^{T} U e_{1}>\left\|C_{1} U e_{1}\right\|$ and $a^{T} U b>\left\|C_{1} U b\right\|$.

For case $(i)$ : It is clear that the corresponding $\left[\begin{array}{c}x^{0} \\ y^{0}\end{array}\right]=0$. Furthermore, since $U^{0}$ is an extreme point of $\mathcal{K}^{\prime}$, the corresponding matrix $Z^{0} \triangleq\left[\begin{array}{cc}X^{0} & \left(W^{0}\right)^{T} \\ W^{0} & Y^{0}\end{array}\right]$ must be an extreme point of

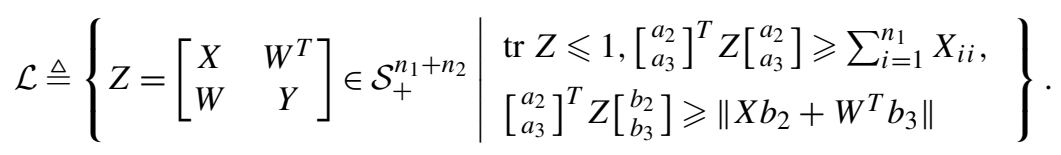

We will discuss three subcases: $Z^{0}\left[\begin{array}{l}b_{2} \\ b_{3}\end{array}\right]=0,\left[\begin{array}{l}a_{2} \\ a_{3}\end{array}\right]^{T} Z^{0}\left[\begin{array}{l}b_{2} \\ b_{3}\end{array}\right]=\left\|X^{0} b_{2}+\left(W^{0}\right)^{T} b_{3}\right\|>0$ and $\left[\begin{array}{l}a_{2} \\ a_{3}\end{array}\right]^{T} Z^{0}\left[\begin{array}{l}b_{2} \\ b_{3}\end{array}\right]>\left\|X^{0} b_{2}+\left(W^{0}\right)^{T} b_{3}\right\|$.

When $Z^{0}\left[\begin{array}{l}b_{2} \\ b_{3}\end{array}\right]=0$, from Proposition 3 of [13], we can always find a rank-one decomposition $Z^{0}=\sum_{i} z^{i}\left(z^{i}\right)^{T}$ satisfying

$$
\left[\begin{array}{l}
a_{2} \\
a_{3}
\end{array}\right]^{T} z^{i}\left(z^{i}\right)^{T}\left[\begin{array}{l}
a_{2} \\
a_{3}
\end{array}\right] \geqslant \sum_{j=1}^{n_{1}}\left(z_{j}^{i}\right)^{2}
$$

Since $Z^{0}$ is positive semidefinite and $\left[\begin{array}{l}b_{2} \\ b_{3}\end{array}\right]^{T} Z^{0}\left[\begin{array}{l}b_{2} \\ b_{3}\end{array}\right]=0$, we have $\left(z^{i}\right)^{T}\left[\begin{array}{l}b_{2} \\ b_{3}\end{array}\right]=0$ for all $i$. One can verify that $Z^{0}=\sum_{i} \frac{\left(z^{i}\right)^{T} z^{i}}{\operatorname{tr} Z^{0}}\left[\frac{\operatorname{tr} Z^{0}}{\left(z^{i}\right)^{T} z^{i}}\left(z^{i}\left(z^{i}\right)^{T}\right)\right]$ and $\frac{\operatorname{tr} Z^{0}}{\left(z^{i}\right)^{T} z^{i}}\left(z^{i}\left(z^{i}\right)^{T}\right) \in \mathcal{L}$ for all $i$. From the fact that $Z^{0}$ is an extreme point of $\mathcal{L}$, then $Z^{0}=\frac{\operatorname{tr} Z^{0}}{\left(z^{i}\right)^{T} z^{i}}\left(z^{i}\left(z^{i}\right)^{T}\right)$ for all $i$, i.e., $\operatorname{rank}\left(Z^{0}\right)=1$. Let $Z^{0}=z^{0}\left(z^{0}\right)^{T}$, then $U^{0}=\left[\begin{array}{c}0 \\ z^{0}\end{array}\right]\left[\begin{array}{c}0 \\ z^{0}\end{array}\right]^{T}$ and $0=a^{T}\left[\begin{array}{c}0 \\ z^{0}\end{array}\right]=$ $b^{T}\left[\begin{array}{c}0 \\ z^{0}\end{array}\right]$. Notice that

$$
0=\left[\begin{array}{l}
a_{2} \\
a_{3}
\end{array}\right]^{T} z^{0}\left(z^{0}\right)^{T}\left[\begin{array}{l}
a_{2} \\
a_{3}
\end{array}\right] \geqslant \sum_{j=1}^{n_{1}}\left(z_{j}^{0}\right)^{2} \geqslant 0
$$

Consequently, $\left[\begin{array}{c}0 \\ z^{0}\end{array}\right] \in \mathcal{H}_{\mathcal{F}}$, i.e., $U^{0} \in \mathcal{D}_{\mathcal{F}}^{*}$.

When $\left[\begin{array}{l}a_{2} \\ a_{3}\end{array}\right]^{T} Z^{0}\left[\begin{array}{l}b_{2} \\ b_{3}\end{array}\right]=\left\|X^{0} b_{2}+\left(W^{0}\right)^{T} b_{3}\right\|>0$, let $z \triangleq Z^{0}\left[\begin{array}{l}b_{2} \\ b_{3}\end{array}\right]$. Noticing that

$$
Z^{0}-\lambda z z^{T}=\left(Z^{0}\right)^{\frac{1}{2}}\left[I-\lambda\left(Z^{0}\right)^{\frac{1}{2}}\left[\begin{array}{l}
b_{2} \\
b_{3}
\end{array}\right]\left[\begin{array}{l}
b_{2} \\
b_{3}
\end{array}\right]^{T}\left(Z^{0}\right)^{\frac{1}{2}}\right]\left(Z^{0}\right)^{\frac{1}{2}}
$$


we know $V \triangleq Z^{0}-\lambda z z^{T}$ is positive semidefinite for some $\lambda>0$. We can rewrite the above equation as

$$
Z^{0}=\frac{\operatorname{tr} V}{\operatorname{tr} Z^{0}}\left(\frac{\operatorname{tr} Z^{0}}{\operatorname{tr} V} V\right)+\frac{\lambda z^{T} z}{\operatorname{tr} Z^{0}}\left(\frac{\operatorname{tr} Z^{0}}{z^{T} z} z z^{T}\right) .
$$

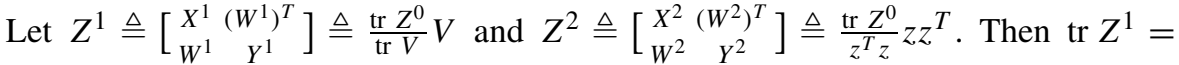
$\operatorname{tr} Z^{2}=\operatorname{tr} Z^{0} \leqslant 1$. One can verify that:

$$
\begin{aligned}
{\left[\begin{array}{l}
a_{2} \\
a_{3}
\end{array}\right]^{T} Z^{2}\left[\begin{array}{l}
a_{2} \\
a_{3}
\end{array}\right] } & =\frac{\operatorname{tr} Z^{0}}{z^{T} z}\left(\left[\begin{array}{l}
a_{2} \\
a_{3}
\end{array}\right]^{T} Z^{0}\left[\begin{array}{l}
b_{2} \\
b_{3}
\end{array}\right]\right)^{2} \\
& =\frac{\operatorname{tr} Z^{0}}{z^{T} z}\left\|X^{0} b_{2}+\left(W^{0}\right)^{T} b_{3}\right\|^{2}=\frac{\operatorname{tr} Z^{0}}{z^{T} z} \sum_{i=1}^{n_{1}} z_{i}^{2}=\sum_{i=1}^{n_{1}}\left[Z^{2}\right]_{i i} .
\end{aligned}
$$

Since $z=Z^{0}\left[\begin{array}{c}b_{2} \\ b_{3}\end{array}\right]=\left[\begin{array}{c}X^{0} b_{2}+\left(W^{0}\right)^{T} b_{3} \\ W^{0} b_{2}+Y^{0} b_{3}\end{array}\right]$ and $Z^{2}\left[\begin{array}{c}b_{2} \\ b_{3}\end{array}\right]=\frac{\operatorname{tr} Z^{0}}{z^{T} z} z^{T}\left[\begin{array}{c}b_{2} \\ b_{3}\end{array}\right] z=\left[\begin{array}{c}X^{2} b_{2}+\left(W^{2}\right)^{T} b_{3} \\ W^{2} b_{2}+Y^{2} b_{3}\end{array}\right]$, we have

$$
\begin{aligned}
{\left[\begin{array}{l}
a_{2} \\
a_{3}
\end{array}\right]^{T} Z^{1}\left[\begin{array}{l}
a_{2} \\
a_{3}
\end{array}\right] } & =\frac{\operatorname{tr} Z^{0}}{\operatorname{tr} V}\left[\begin{array}{l}
a_{2} \\
a_{3}
\end{array}\right]^{T}\left(Z^{0}-\lambda z z^{T}\right)\left[\begin{array}{l}
a_{2} \\
a_{3}
\end{array}\right] \geqslant \frac{\operatorname{tr} Z^{0}}{\operatorname{tr} V} \sum_{i=1}^{n_{1}}\left(X_{i i}^{0}-\lambda z_{i}^{2}\right) \\
& =\frac{\operatorname{tr} Z^{0}}{\operatorname{tr} V} \sum_{i=1}^{n_{1}} V_{i i}=\sum_{i=1}^{n_{1}} Z_{i i}^{1}, \\
{\left[\begin{array}{l}
a_{2} \\
a_{3}
\end{array}\right]^{T} Z^{2}\left[\begin{array}{l}
b_{2} \\
b_{3}
\end{array}\right] } & =\frac{\operatorname{tr} Z^{0}}{z^{T} z}\left[\begin{array}{l}
a_{2} \\
a_{3}
\end{array}\right]^{T} Z^{0}\left[\begin{array}{l}
b_{2} \\
b_{3}
\end{array}\right]\left[\begin{array}{l}
b_{2} \\
b_{3}
\end{array}\right]^{T} Z^{0}\left[\begin{array}{l}
b_{2} \\
b_{3}
\end{array}\right] \\
& =\frac{\operatorname{tr} Z^{0}}{z^{T} z}\left\|X^{0} b_{2}+\left(W^{0}\right)^{T} b_{3}\right\|\left(z^{T}\left[\begin{array}{l}
b_{2} \\
b_{3}
\end{array}\right]\right) \\
& =\left\|X^{2} b_{2}+\left(W^{2}\right)^{T} b_{3}\right\|,
\end{aligned}
$$

and

$$
\begin{aligned}
{\left[\begin{array}{l}
a_{2} \\
a_{3}
\end{array}\right]^{T} Z^{1}\left[\begin{array}{l}
b_{2} \\
b_{3}
\end{array}\right] } & =\frac{\operatorname{tr} Z^{0}}{\operatorname{tr} V}\left[\begin{array}{l}
a_{2} \\
a_{3}
\end{array}\right]^{T}\left(Z^{0}-\lambda z z^{T}\right)\left[\begin{array}{l}
b_{2} \\
b_{3}
\end{array}\right] \\
& =\frac{\operatorname{tr} Z^{0}}{\operatorname{tr} V}\left\|X^{0} b_{2}+\left(W^{0}\right)^{T} b_{3}\right\|\left(1-\lambda\left[\begin{array}{l}
b_{2} \\
b_{3}
\end{array}\right]^{T} Z^{0}\left[\begin{array}{l}
b_{2} \\
b_{3}
\end{array}\right]\right) \\
& =\left\|X^{1} b_{2}+\left(W^{1}\right)^{T} b_{3}\right\| .
\end{aligned}
$$


Therefore, $Z^{1}$ and $Z^{2}$ are all in $\mathcal{L}$. Since $Z^{0}$ is an extreme point of $\mathcal{L}$, then $Z^{0}=Z^{1}=$ $Z^{2}$, i.e., $Z^{0}=\frac{\operatorname{tr} Z^{0}}{z^{T} z} z z^{T}$. Let $u^{0} \triangleq\left[\begin{array}{c}0 \\ u_{x}^{0} \\ u_{y}^{0}\end{array}\right] \triangleq\left[\frac{0}{\sqrt{\frac{\operatorname{tr}\left(Z^{0}\right)}{z^{T} z}}}\right]$, then $U^{0}=u^{0}\left(u^{0}\right)^{T}$. Notice that

$$
\begin{aligned}
b^{T} u^{0} & =a^{T} u^{0}=a_{2}^{T} u_{x}^{0}+a_{3}^{T} u_{y}^{0}=\sqrt{\frac{\operatorname{tr} Z^{0}}{z^{T} z}}\left[\begin{array}{l}
a_{2} \\
a_{3}
\end{array}\right]^{T} Z^{0}\left[\begin{array}{l}
b_{2} \\
b_{3}
\end{array}\right] \\
& =\sqrt{\frac{\operatorname{tr} Z^{0}}{z^{T} z}}\left\|X^{0} b_{2}+\left(W^{0}\right)^{T} b_{3}\right\|=\left\|u_{x}^{0}\right\| .
\end{aligned}
$$

Consequently, we have $u^{0} \in \mathcal{H}_{\mathcal{F}}$ and $U^{0} \in \mathcal{D}_{\mathcal{F}}^{*}$.

When $\left[\begin{array}{l}a_{2} \\ a_{3}\end{array}\right]^{T} Z^{0}\left[\begin{array}{l}b_{2} \\ b_{3}\end{array}\right]>\left\|X^{0} b_{2}+\left(W^{0}\right)^{T} b_{3}\right\|$, we know that $\left(Z^{0}, S^{0}, s_{1}^{0}, s_{2}^{0}\right)$, where

$$
\begin{aligned}
& S^{0} \triangleq \operatorname{Arrow}\left(\left[\begin{array}{l}
a_{2} \\
a_{3}
\end{array}\right]^{T} Z^{0}\left[\begin{array}{l}
b_{2} \\
b_{3}
\end{array}\right], X^{0} b_{2}+\left(W^{0}\right)^{T} b_{3}\right), \\
& s_{1}^{0} \triangleq 1-\operatorname{tr} Z^{0} \quad \text { and } \quad s_{2}^{0} \triangleq\left[\begin{array}{l}
a_{2} \\
a_{3}
\end{array}\right]^{T} Z^{0}\left[\begin{array}{l}
a_{2} \\
a_{3}
\end{array}\right]-\sum_{i=1}^{n_{1}} X_{i i}^{0},
\end{aligned}
$$

is an extreme point of

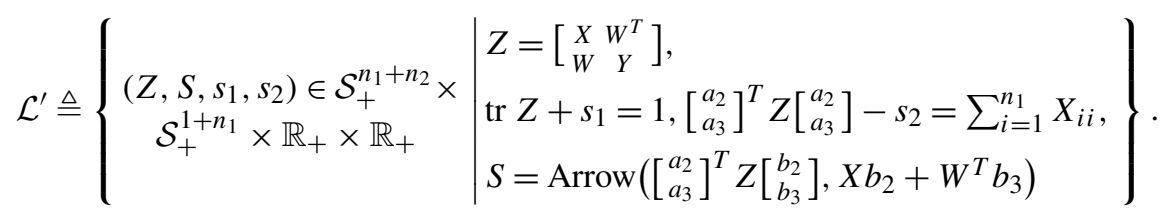

From Lemma 9, let $r_{Z} \triangleq \operatorname{rank}\left(Z^{0}\right), r_{S} \triangleq \operatorname{rank}\left(S^{0}\right), r_{1} \triangleq \operatorname{rank}\left(s_{1}^{0}\right), r_{2} \triangleq \operatorname{rank}\left(s_{2}^{0}\right)$. Then

$$
r_{Z}\left(r_{Z}+1\right)+r_{S}\left(r_{S}+1\right)+r_{1}\left(r_{1}+1\right)+r_{2}\left(r_{2}+1\right) \leqslant 4+\left(n_{1}+1\right)\left(n_{1}+2\right) .
$$

Since $r_{S}=n_{1}+1$, by Lemma 10, we have $r_{Z}=1$ and $Z^{0}=\left[\begin{array}{l}x^{\prime} \\ y^{\prime}\end{array}\right]\left[\begin{array}{l}x^{\prime} \\ y^{\prime}\end{array}\right]^{T}$ with $a_{2}^{T} x^{\prime}+$ $a_{3}^{T} y^{\prime} \geqslant\left\|x^{\prime}\right\|$. Consequently, $U^{0}=\left[\begin{array}{c}0 \\ x^{\prime} \\ y^{\prime}\end{array}\right]\left[\begin{array}{c}0 \\ x^{\prime} \\ y^{\prime}\end{array}\right]^{T}$. Noticing that $b^{T}\left[\begin{array}{c}0 \\ x^{\prime} \\ y^{\prime}\end{array}\right]=a^{T}\left[\begin{array}{c}0 \\ x^{\prime} \\ y^{\prime}\end{array}\right] \geqslant$ $\left\|x^{\prime}\right\|$, we have $\left[\begin{array}{c}0 \\ x^{\prime} \\ y^{\prime}\end{array}\right] \in \mathcal{H}_{\mathcal{F}}$ and $U^{0} \in \mathcal{D}_{\mathcal{F}}^{*}$.

Therefore, we have shown that our claim holds for case (i).

For case (ii): Since $\chi>0$, we have $U^{0} e_{1} \neq 0$. Define $U^{1} \triangleq \frac{U^{0} e_{1} e_{1}^{T} U^{0}}{e_{1}^{T} U^{0} e_{1}}$ and $U^{2} \triangleq$ $U^{0}-U^{1}$. From Lemma 11, we know $U^{2}$ is positive semidefinite.

When $U^{2} a=0$, we have $0=U^{2} a=U^{0} a-\frac{e_{1}^{T} U^{0} a}{e_{1}^{T} U^{0} e_{1}} U^{0} e_{1}$, i.e., $U^{0} a$ and $U^{0} e_{1}$ are linearly dependent. Therefore, $U^{0} b=U^{0} a-a_{4} U^{0} e_{1}$ is also linearly dependent on 
$U^{0} e_{1}$. Rewrite

$$
U^{0}=\frac{\operatorname{tr} U^{1}}{\operatorname{tr} U^{0}}\left(\frac{\operatorname{tr} U^{0}}{\operatorname{tr} U^{1}} U^{1}\right)+\frac{\operatorname{tr} U^{2}}{\operatorname{tr} U^{0}}\left(\frac{\operatorname{tr} U^{0}}{\operatorname{tr} U^{2}} U^{2}\right)
$$

and one can verify that $\frac{\operatorname{tr} U^{0}}{\operatorname{tr} U^{1}} U^{1} \in \mathcal{K}^{\prime}$. To see $\frac{\operatorname{tr} U^{0}}{\operatorname{tr} U^{2}} U^{2} \in \mathcal{K}^{\prime}$, we only need to show $U^{2} \in \mathcal{K}$. From $U^{2} a=U^{2} e_{1}=0$, we have $a^{T} U^{2} e_{1} \geqslant\left\|C_{1} U^{2} e_{1}\right\|$ and $b^{T} U^{2} e_{1} \geqslant 0$. Notice that

$$
U^{2} \bullet\left(a a^{T}-C_{1}^{T} C_{1}\right)=\left(U^{0}-U^{1}\right) \bullet\left(a a^{T}-C_{1}^{T} C_{1}\right)=U^{0} \bullet\left(a a^{T}-C_{1}^{T} C_{1}\right) \geqslant 0 .
$$

From $U^{2} a=0$, we have $\operatorname{tr}\left(C_{1} U^{2} C_{1}^{T}\right)=0$. Consequently, $C_{1} U^{2}=0, a^{T} U^{2} b=0$ and $C_{1} U^{2} b=0$. Hence $U^{2} \in \mathcal{K}$ and $\frac{\operatorname{tr} U^{0}}{\operatorname{tr} U^{2}} U^{2} \in \mathcal{K}^{\prime}$. Since $U^{0}$ is an extreme point in $\mathcal{K}^{\prime}$, either $U^{2}=0$ or $U^{0}=\frac{\operatorname{tr} U^{0}}{\operatorname{tr} U^{1}} U^{1}=\frac{\operatorname{tr} U^{0}}{\operatorname{tr} U^{2}} U^{2}$. Noticing that $U^{1} e_{1}=U^{0} e_{1} \neq 0=$ $U^{2} e_{1}$, we must have $U^{2}=0$ and $U^{0}=U^{1}=\frac{U^{0} e_{1} e_{1}^{T} U^{0}}{e_{1}^{T} U^{0} e_{1}}$. From $U^{0} \in \mathcal{K}$, one can verify that $\frac{U^{0} e_{1}}{\sqrt{e_{1}^{T} U^{0} e_{1}}}$ is in $\mathcal{H}_{\mathcal{F}}$ and, therefore, $U^{0} \in \mathcal{D}_{\mathcal{F}}^{*}$.

When $U^{2} a \neq 0$, we have $a^{T} U^{2} a \neq 0$. Let $U^{3} \triangleq \frac{U^{2} a a^{T} U^{2}}{a^{T} U^{2} a}$ and $U^{4} \triangleq U^{2}-U^{3}=$ $U^{0}-U^{1}-U^{3}$. From Lemma 11 , we know $U^{3}$ and $U^{4}$ are both positive semidefinite.

From $\operatorname{tr}\left(C_{1} U^{4} C_{1}^{T}\right) \geqslant 0$, we have

$$
\begin{aligned}
&\left(a^{T} U^{2} a\right)\left[\operatorname{tr}\left(C_{1} U^{0} C_{1}^{T}\right)-a^{T} U^{0} a\right] \\
& \geqslant\left(a^{T} U^{2} a\right)\left[\operatorname{tr}\left(C_{1}\left(U^{1}+U^{3}\right) C_{1}^{T}\right)-a^{T} U^{0} a\right] \\
&=\left(a^{T} U^{2} a\right) \frac{\left\|C_{1} U^{0} e_{1}\right\|^{2}}{e_{1}^{T} U^{0} e_{1}}+\left\|C_{1} U^{0} a-\frac{e_{1}^{T} U^{0} a}{e_{1}^{T} U^{0} e_{1}} C_{1} U^{0} e_{1}\right\|^{2} \\
&-\left(a^{T} U^{2} a\right)\left(a^{T} U^{0} a\right) \\
&= \frac{a^{T} U^{0} a\left\|C_{1} U^{0} e_{1}\right\|^{2}}{e_{1}^{T} U^{0} e_{1}}+\left\|C_{1} U^{0} a\right\|^{2}-\frac{2 e_{1}^{T} U^{0} a}{e_{1}^{T} U^{0} e_{1}} e_{1}^{T} U^{0} C_{1}^{T} C_{1} U^{0} a \\
&-\left(a^{T} U^{2} a\right)\left(a^{T} U^{0} a\right) \\
&= 2 \frac{\left(a^{T} U^{0} a\right)\left(e_{1}^{T} U^{0} a\right)^{2}}{e_{1}^{T} U^{0} e_{1}}+\left\|C_{1} U^{0} a\right\|^{2}-\frac{2 e_{1}^{T} U^{0} a}{e_{1}^{T} U^{0} e_{1}} e_{1}^{T} U^{0} C_{1}^{T} C_{1} U^{0} a-\left(a^{T} U^{0} a\right)^{2} \\
&= 2 \frac{\left(a^{T} U^{0} a\right)\left(e_{1}^{T} U^{0} a\right)^{2}}{e_{1}^{T} U^{0} e_{1}}-\frac{2 e_{1}^{T} U^{0} a}{e_{1}^{T} U^{0} e_{1}} e_{1}^{T} U^{0} C_{1}^{T} C_{1} U^{0} a+\left\|C_{1} U^{0} b+a_{4} C_{1} U^{0} e_{1}\right\|^{2} \\
&-\left(a^{T} U^{0} b+a_{4} a^{T} U^{0} e_{1}\right)^{2} \\
&= 2 \frac{\left(a^{T} U^{0} a\right)\left(e_{1}^{T} U^{0} a\right)^{2}}{e_{1}^{T} U^{0} e_{1}}-\frac{2 e_{1}^{T} U^{0} a}{e_{1}^{T} U^{0} e_{1}} e_{1}^{T} U^{0} C_{1}^{T} C_{1} U^{0} a \\
&+2 a_{4} e_{1}^{T} U^{0} C_{1}^{T} C_{1} U^{0}\left(a-a_{4} e_{1}\right)-2 a_{4}\left(a^{T} U^{0} e_{1}\right) a^{T} U^{0}\left(a-a_{4} e_{1}\right)
\end{aligned}
$$




$$
\begin{aligned}
& =2 \frac{\left(a^{T} U^{0} a\right)\left(e_{1}^{T} U^{0} a\right) e_{1}^{T} U^{0}\left(a-a_{4} e_{1}\right)}{e_{1}^{T} U^{0} e_{1}}-\frac{2 e_{1}^{T} U^{0}\left(a-a_{4} e_{1}\right)}{e_{1}^{T} U^{0} e_{1}} e_{1}^{T} U^{0} C_{1}^{T} C_{1} U^{0} a \\
& =2 \frac{e_{1}^{T} U^{0} b}{e_{1}^{T} U^{0} e_{1}}\left(\left(a^{T} U^{0} a\right)\left(e_{1}^{T} U^{0} a\right)-e_{1}^{T} U^{0} C_{1}^{T} C_{1} U^{0} a\right) \geqslant 0 .
\end{aligned}
$$

The above inequality indicates that $\operatorname{tr}\left(C_{1} U^{0} C_{1}^{T}\right)=a^{T} U^{0} a$ if and only if $\operatorname{tr}\left(C_{1} U^{4} C_{1}^{T}\right)=0$ and $\left[\begin{array}{l}a^{T} U^{0} e_{1} \\ C_{1} U^{0} e_{1}\end{array}\right]$ and $\left[\begin{array}{c}a^{T} U^{0} a \\ C_{1} U^{0} a\end{array}\right]$ are linearly dependent. Consequently, when $\operatorname{tr}\left(C_{1} U^{0} C_{1}^{T}\right)=a^{T} U^{0} a$, we know that $\left[\begin{array}{c}a^{T} U^{0} e_{1} \\ C_{1} U^{0} e_{1}\end{array}\right]$ and $\left[\begin{array}{c}a^{T} U^{0} b \\ C_{1} U^{0} b\end{array}\right]$ are linearly dependent. Notice that

$$
a^{T} U^{2} b=a^{T} U^{2} a-a_{4} a^{T} U^{2} e_{1}=a^{T} U^{2} a>0
$$

and $\left[\begin{array}{l}a^{T} U^{2} b \\ C_{1} U^{2} b\end{array}\right]=\left[\begin{array}{l}a^{T} U^{0} b \\ C_{1} U^{0} b\end{array}\right]-\frac{e_{1}^{T} U^{0} b}{e_{1}^{T} U^{0} e_{1}}\left[\begin{array}{l}a^{T} U^{0} e_{1} \\ C_{1} U^{0} e_{1}\end{array}\right]$. Hence $a^{T} U^{2} b=\left\|C_{1} U^{2} b\right\|$. Then we can easily verify that $U^{1}$ and $U^{2}$ are both in $\mathcal{K}$. From

$$
U^{0}=\frac{\operatorname{tr} U^{1}}{\operatorname{tr} U^{0}}\left(\frac{\operatorname{tr} U^{0}}{\operatorname{tr} U^{1}} U^{1}\right)+\frac{\operatorname{tr} U^{2}}{\operatorname{tr} U^{0}}\left(\frac{\operatorname{tr} U^{0}}{\operatorname{tr} U^{2}} U^{2}\right)
$$

we know $\frac{\operatorname{tr} U^{0}}{\operatorname{tr} U^{1}} U^{1}$ and $\frac{\operatorname{tr} U^{0}}{\operatorname{tr} U^{2}} U^{2}$ are both in $\mathcal{K}^{\prime}$. Therefore, $U^{0}=U^{1}=\frac{U^{0} e_{1} e_{1}^{T} U^{0}}{e_{1}^{T} U^{0} e_{1}}$. From $U^{0} \in \mathcal{K}$, one can verify that $\frac{U^{0} e_{1}}{\sqrt{e_{1}^{T} U^{0} e_{1}}} \in \mathcal{H}_{\mathcal{F}}$ and $U^{0} \in \mathcal{D}_{\mathcal{F}}^{*}$.

Hence we have shown that $U^{0} \in \mathcal{D}_{\mathcal{F}}^{*}$ in case (ii).

For case (iii): We let $U^{1} \triangleq \lambda U^{0} b b^{T} U^{0}$ and $U^{2} \triangleq U^{0}-U^{1}$ with $\lambda>0$ being a sufficiently small number. One can easily check that $U^{1} \in \mathcal{K}$. When $\lambda$ is small enough, $U^{2}$ is positive semidefinite. From $a^{T} U^{0} e_{1}>\left\|C_{1} U^{0} e_{1}\right\|$, we know that $\left[\begin{array}{l}a^{T} U^{0} e_{1} \\ C_{1} U^{0} e_{1}\end{array}\right]$ is an interior point of $\mathcal{S O C}\left(n_{1}\right)$. Therefore, $\left[\begin{array}{c}a^{T} U^{2} e_{1} \\ C_{1} U^{2} e_{1}\end{array}\right]=\left[\begin{array}{l}a^{T} U^{0} e_{1} \\ C_{1} U^{0} e_{1}\end{array}\right]-\lambda\left(b^{T} U^{0} e_{1}\right)\left[\begin{array}{c}a^{T} U^{0} b \\ C_{1} U^{0} b\end{array}\right]$ is also in $\mathcal{S O C}\left(n_{1}\right)$ when $\lambda$ is small enough, i.e., $a^{T} U^{2} e_{1} \geqslant\left\|C_{1} U^{2} e_{1}\right\|$. We can also see that

$$
\begin{aligned}
& U^{2} \bullet\left(a a^{T}-C_{1}^{T} C_{1}\right)=\left(U^{0}-U^{1}\right) \bullet\left(a a^{T}-C_{1}^{T} C_{1}\right)=U^{0} \bullet\left(a a^{T}-C_{1}^{T} C_{1}\right) \geqslant 0, \\
& b^{T} U^{2} e_{1}=b^{T} U^{0} e_{1}-\lambda\left(b^{T} U^{0} b\right) b^{T} U^{0} e_{1} \geqslant 0, \quad \text { and } \\
& a^{T} U^{2} b=\left(1-\lambda\left(b^{T} U^{0} b\right)\right) a^{T} U^{0} b \geqslant\left\|\left(1-\lambda\left(b^{T} U^{0} b\right)\right) C_{1} U^{0} b\right\|=\left\|C_{1} U^{2} b\right\| .
\end{aligned}
$$

Therefore, we have $U^{2} \in \mathcal{K}$. Since $U^{0}$ is an extreme point of $\mathcal{K}^{\prime}$, we know $U^{0}=$ $\frac{\operatorname{tr} U^{0}}{\operatorname{tr} U^{1}} U^{1}=\frac{\operatorname{tr} U^{0}}{\operatorname{tr} U^{2}} U^{2}$. However, $a^{T} U^{0} e_{1}=\frac{\operatorname{tr} U^{0}}{\operatorname{tr} U^{1}} a^{T} U^{1} e_{1}=\frac{\operatorname{tr} U^{0}}{\operatorname{tr} U^{1}} \lambda\left(b^{T} U^{0} e_{1}\right)\left(a^{T} U^{0} b\right) \times$ $=\frac{\operatorname{tr} U^{0}}{\operatorname{tr} U^{1}} \lambda\left(b^{T} U^{0} e_{1}\right)\left\|C_{1} U^{0} b\right\|=\frac{\operatorname{tr} U^{0}}{\operatorname{tr} U^{1}}\left\|C_{1} U^{1} e_{1}\right\|=\left\|C_{1} U^{0} e_{1}\right\|$, which contradicts to $a^{T} U^{0} e_{1}>\left\|C_{1} U^{0} e_{1}\right\|$. This shows that no extreme point of $\mathcal{K}^{\prime}$ exists in case (iii).

For case (iv): The proof is similar to that of case (iii). 
For case (v): Let

$$
\mathcal{L}^{\prime \prime} \triangleq\left\{\begin{array}{l|l}
\left(U, S_{1}, S_{2}, s_{1}, s_{2}, s_{3}\right) \in & U=\left[\begin{array}{ccc}
x & x^{T} & y^{T} \\
x & x & W^{T} \\
y & W & Y
\end{array}\right], \\
\mathcal{S}_{+}^{1+n_{1}+n_{2}} \times \mathcal{S}_{+}^{1+n_{1}} \times \mathcal{S}_{+}^{1+n_{1}} \times & \begin{array}{l}
S_{1}=\operatorname{Arrow}\left(a^{T} U e_{1}, C_{1} U e_{1}\right), \\
S_{2}=\operatorname{Arrow}\left(a^{T} U b, C_{1} U b\right), \\
\mathbb{R}_{+} \times \mathbb{R}_{+} \times \mathbb{R}_{+} \\
s_{1}=U \bullet\left(a a^{T}-C_{1}^{T} C_{1}\right), \\
s_{2}=b^{T} U e_{1}, s_{3}=1-\operatorname{tr} U
\end{array}
\end{array}\right\} .
$$

Since $U^{0}$ is an extreme point of $\mathcal{K}^{\prime}$, we can see the corresponding $\left(U^{0}, S_{1}^{0}, S_{2}^{0}, s_{1}^{0}\right.$, $\left.s_{2}^{0}, s_{3}^{0}\right)$ is an extreme point of $\mathcal{L}^{\prime \prime}$. From Lemma 9, let $r_{U} \triangleq \operatorname{rank}\left(U^{0}\right), r_{S_{1}} \triangleq$ $\operatorname{rank}\left(S_{1}^{0}\right), r_{S_{2}} \triangleq \operatorname{rank}\left(S_{2}^{0}\right), r_{1} \triangleq \operatorname{rank}\left(s_{1}^{0}\right), r_{2} \triangleq \operatorname{rank}\left(s_{2}^{0}\right)$ and $r_{3} \triangleq \operatorname{rank}\left(s_{3}^{0}\right)$, then we have

$$
\begin{aligned}
& r_{U}\left(r_{U}+1\right)+r_{S_{1}}\left(r_{S_{1}}+1\right)+r_{S_{2}}\left(r_{S_{2}}+1\right)+r_{1}\left(r_{1}+1\right)+r_{2}\left(r_{2}+1\right)+r_{3}\left(r_{3}+1\right) \\
& \quad \leqslant 2\left(n_{1}+1\right)\left(n_{1}+2\right)+6 .
\end{aligned}
$$

Based on the assumption for (v), we have $a^{T} U^{0} e_{1}>\left\|C_{1} U^{0} e_{1}\right\|$ and $a^{T} U^{0} b>$ $\left\|C_{1} U^{0} b\right\|$. Lemma 10 implies that $r_{S_{1}}=r_{S_{2}}=1+n_{1}$. Then the above inequality becomes

$$
r_{U}\left(r_{U}+1\right)+r_{1}\left(r_{1}+1\right)+r_{2}\left(r_{2}+1\right)+r_{3}\left(r_{3}+1\right) \leqslant 6 .
$$

If $r_{U}=1$, then one can easily verify that $U^{0} \in \mathcal{D}_{\mathcal{F}}^{*}$. If $r_{U}=2$, we show that $U^{0}$ cannot be an extreme point of $\mathcal{K}^{\prime}$. In this situation, $r_{1}=r_{2}=r_{3}=0$, i.e., $s_{1}^{0}=s_{2}^{0}=$ $s_{3}^{0}=0$. From $a^{T} U^{0} e_{1}>0$ and $a^{T} U^{0} b>0$, we have $U^{0} e_{1} \neq 0$ and $U^{0} b \neq 0$. Define $U^{1} \triangleq \frac{U^{0} e_{1} e_{1}^{T} U^{0}}{e_{1}^{T} U^{0} e_{1}}$ and $U^{2} \triangleq U^{0}-U^{1}$. From Lemma $11, U^{2}$ is positive semidefinite and $\operatorname{rank}\left(U^{2}\right)=1$. Since $s_{2}^{0}=b^{T} U^{0} e_{1}=0$, we have $U^{2} b=U^{0} b \neq 0$. Therefore, $U^{2}=\frac{U^{2} b b^{T} U^{2}}{b^{T} U^{2} b}=\frac{U^{0} b b^{T} U^{0}}{b^{T} U^{0} b}$. This means $U^{0}=\frac{U^{0} e_{1} e_{1}^{T} U^{0}}{e_{1}^{T} U^{0} e_{1}}+\frac{U^{0} b b^{T} U^{0}}{b^{T} U^{0} b}$. Notice that $U^{0} b$ and $U^{0} e_{1}$ are linearly independent. (Otherwise, $0 \neq b^{T} U^{0} b=\tau b^{T} U^{0} e_{1}=0$ for some $\tau \neq 0$, which causes a contradiction.) One can further verify that $\frac{\operatorname{tr} U^{0}}{\operatorname{tr} U^{1}} U^{1}$ and $\frac{\operatorname{tr} U^{0}}{\operatorname{tr} U^{2}} U^{2}$ are all in $\mathcal{K}^{\prime}$ and $U^{0}$ is the convex combination of these two distinct points which means $U^{0}$ cannot be an extreme point of $\mathcal{K}^{\prime}$.

From the discussion of the above five cases, we have $\mathcal{K} \subseteq \mathcal{D}_{\mathcal{F}}^{*}$ and hence $\mathcal{K}=\mathcal{D}_{\mathcal{F}}^{*}$.

We now prove the dual part. Notice that

$$
\begin{aligned}
\mathcal{D}_{\mathcal{F}}^{*}= & \mathcal{S}_{+}^{1+n_{1}+n_{2}} \cap\left\{U \mid a^{T} U e_{1} \geqslant\left\|C_{1} U e_{1}\right\|\right\} \cap\left\{U \mid U \bullet\left(a a^{T}-C_{1}^{T} C_{1}\right) \geqslant 0\right\} \\
& \cap\left\{U \mid b^{T} U e_{1} \geqslant 0\right\} \cap\left\{U \mid a^{T} U b \geqslant\left\|C_{1} U b\right\|\right\} .
\end{aligned}
$$

From Lemma 5, its dual is

$$
\mathcal{D}_{\mathcal{F}}=\operatorname{cl}\left(\begin{array}{c}
\mathcal{S}_{+}^{1+n_{1}+n_{2}}+\left\{U \mid a^{T} U e_{1} \geqslant\left\|C_{1} U e_{1}\right\|\right\}^{*} \\
+\left\{U \mid U \bullet\left(a a^{T}-C_{1}^{T} C_{1}\right) \geqslant 0\right\}^{*} \\
\quad+\left\{U \mid b^{T} U e_{1} \geqslant 0\right\}^{*}+\left\{U \mid a^{T} U b \geqslant\left\|C_{1} U b\right\|\right\}^{*}
\end{array}\right)
$$




$$
=\operatorname{cl}\left\{\begin{array}{l|l}
M \in \mathcal{S}^{1+n_{1}+n_{2}} & \begin{array}{l}
M-\lambda_{1} C_{3}-\lambda_{2}\left(e_{1} b^{T}+b e_{1}^{T}\right)-\left(e_{1} \psi_{1}^{T} C_{2}^{T}+C_{2} \psi_{1} e_{1}^{T}\right) \\
-\left(b \psi_{2}^{T} C_{2}^{T}+C_{2} \psi_{2} b^{T}\right) \in \mathcal{S}_{+}^{1+n_{1}+n_{2}} \\
\lambda_{1}, \lambda_{2} \geqslant 0, \psi_{1}, \psi_{2} \in \mathcal{S O C}\left(n_{1}\right)
\end{array}
\end{array}\right\} .
$$

Then it follows from Sturm and Zhang [13] that QCQP, LCoP and LCoD all have the same optimal value.

We now prove the second half of the theorem. If there is $(\bar{x}, \bar{y}) \in \mathbb{R}^{n_{1}} \times \mathbb{R}^{n_{2}}$ such that $\|\bar{x}\|<a_{1}+a_{2}^{T} \bar{x}+a_{3}^{T} \bar{y}$ and $a_{1}+a_{2}^{T} \bar{x}+a_{3}^{T} \bar{y}>a_{4}$, then let $\bar{u} \triangleq\left[\begin{array}{c}1 \\ \bar{x} \\ \bar{y}\end{array}\right]$ and $\bar{U} \triangleq$ $\bar{u} \bar{u}^{T}$. In this way, $\bar{U} \in \mathcal{S}_{+}^{1+n_{1}+n_{2}}, C_{2} \bar{U} e_{1} \in \operatorname{int} \mathcal{S O C}\left(n_{1}\right), \bar{U} \bullet C_{3}>0, b^{T} \bar{U} e_{1}>0$ and $C_{2} \bar{U} b \in \operatorname{int} \mathcal{S O C}\left(n_{1}\right)$. Let $U^{\prime} \triangleq \bar{U}+\tau I_{1+n_{1}+n_{2}}, \tau>0$. When $\tau$ is sufficiently small, we know $U^{\prime}$ is an interior point of $\mathcal{D}_{\mathcal{F}}^{*}$. Therefore, using Lemma 5, the closure can be removed from $\mathcal{D}_{\mathcal{F}}$ and the rest of the claims becomes true.

Remark 5 From the above proof, we see that an optimal extreme solution of the problem LCoP can lead to an optimal solution of the original problem QCQP through the explicit rank-one decomposition. Hence we have an exact solvable representation of the QCQP problem whose domain is defined by one second-order cone constraint and one special linear constraint.

When $a_{4}=0$, Theorem 1 can be simplified as follows.

Corollary 1 Given a nonempty set $\mathcal{F}=\left\{(x, y) \in \mathbb{R}^{n_{1}} \times \mathbb{R}^{n_{2}} \mid\|x\| \leqslant a_{1}+a_{2}^{T} x+a_{3}^{T} y\right\}$ with $a_{1} \in \mathbb{R}, a_{2} \in \mathbb{R}^{n_{1}}, a_{3} \in \mathbb{R}^{n_{2}}$ and $a_{4}=0$. Let

$$
\begin{aligned}
& a^{T} \triangleq\left[\begin{array}{lll}
a_{1} & a_{2}^{T} & a_{3}^{T}
\end{array}\right], \\
& C_{1} \triangleq\left[\begin{array}{lll}
0 & I_{n_{1}} & 0
\end{array}\right], \\
& e_{1} \triangleq(1,0, \cdots, 0)^{T} \in \mathbb{R}^{1+n_{1}+n_{2}}, \\
& C_{2} \triangleq\left[\begin{array}{ll}
a & C_{1}^{T}
\end{array}\right] \quad \text { and } \\
& C_{3} \triangleq a a^{T}-C_{1}^{T} C_{1} .
\end{aligned}
$$

Then we have

$$
\begin{gathered}
\mathcal{D}_{\mathcal{F}}^{*}=\left\{U=\left[\begin{array}{ccc}
\chi & x^{T} & y^{T} \\
x & X & W^{T} \\
y & W & Y
\end{array}\right] \in \mathcal{S}_{+}^{1+n_{1}+n_{2}} \mid\right. \\
\left.\left\|C_{1} U e_{1}\right\| \leqslant a^{T} U e_{1}, U \bullet\left(a a^{T}-C_{1}^{T} C_{1}\right) \geqslant 0,\right\}, \\
\mathcal{D}_{\mathcal{F}}=\operatorname{cl}\left\{M \in \mathcal{S}^{1+n_{1}+n_{2}} \mid M-\lambda C_{3}-\left(e_{1} \psi^{T} C_{2}^{T}+C_{2} \psi e_{1}^{T}\right) \in \mathcal{S}_{+}^{1+n_{1}+n_{2}},\right. \\
\left.\lambda \geqslant 0, \psi \in \mathcal{S O C}\left(n_{1}\right)\right\} .
\end{gathered}
$$

Moreover, the corresponding QCQP and LCoP have the same optimal value. 
If there exists $(\bar{x}, \bar{y}) \in \mathbb{R}^{n_{1}} \times \mathbb{R}^{n_{2}}$ such that $\|\bar{x}\|<a_{1}+a_{2}^{T} \bar{x}+a_{3}^{T} \bar{y}$, then we have

$$
\begin{gathered}
\mathcal{D}_{\mathcal{F}}=\left\{M \in \mathcal{S}^{1+n_{1}+n_{2}} \mid M-\lambda C_{3}-\left(e_{1} \psi^{T} C_{2}^{T}+C_{2} \psi e_{1}^{T}\right) \in \mathcal{S}_{+}^{1+n_{1}+n_{2}},\right. \\
\left.\lambda \geqslant 0, \psi \in \mathcal{S O C}\left(n_{1}\right)\right\} .
\end{gathered}
$$

Moreover, the corresponding problem LCoD

$\sup \sigma$

$$
\begin{array}{ll}
\text { s.t. } & {\left[\begin{array}{cc}
c_{0} & b_{0}^{T} \\
b_{0} & A_{0}
\end{array}\right]-\left[\begin{array}{cc}
\sigma & 0 \\
0 & 0
\end{array}\right]-\lambda C_{3}-\left(e_{1} \psi^{T} C_{2}^{T}+C_{2} \psi e_{1}^{T}\right) \in \mathcal{S}_{+}^{1+n_{1}+n_{2}}} \\
& \sigma \in \mathbb{R}, \lambda \geqslant 0, \psi \in \mathcal{S O C}\left(n_{1}\right)
\end{array}
$$

attains the same optimal value as that of the original QCQP.

Proof It is sufficient to show that the three constraints in $\mathcal{D}_{\mathcal{F}}^{*}$ of this corollary imply the five constraints in the $\mathcal{D}_{\mathcal{F}}^{*}$ of Theorem 1 when $a_{4}=0$. Let $b \triangleq a-a_{4} e_{1}=a$. From $U \bullet\left(a a^{T}-C_{1}^{T} C_{1}\right) \geqslant 0$ and

$$
\left[\begin{array}{l}
a^{T} \\
C_{1}
\end{array}\right] U\left[\begin{array}{ll}
a & C_{1}^{T}
\end{array}\right]=\left[\begin{array}{ll}
a^{T} U a & a^{T} U C_{1}^{T} \\
C_{1} U a & C_{1} U C_{1}^{T}
\end{array}\right] \in \mathcal{S}_{+}^{1+n_{1}},
$$

we have $\left(a^{T} U a\right)^{2} \geqslant\left(a^{T} U a\right) \operatorname{tr}\left(C_{1} U C_{1}^{T}\right) \geqslant \operatorname{tr}\left(C_{1} U a a^{T} U C_{1}^{T}\right)=\left\|C_{1} U a\right\|^{2}$. This shows $a^{T} U b \geqslant\left\|C_{1} U b\right\|$. The constraint of $b^{T} U e_{1} \geqslant 0$ is obvious. Therefore, all the five constraints in the $\mathcal{D}_{\mathcal{F}}^{*}$ of Theorem 1 are satisfied.

Notice that the domain $\mathcal{F}$ defined in Theorem 1 is an unbounded set. The next theorem provides an exact computable representation of the QCQP problem whose domain consists of one second-order cone constraint with both lower and upper bounds.

Theorem 2 Given a nonempty set $\mathcal{F}=\left\{(x, y) \in \mathbb{R}^{n_{1}} \times \mathbb{R}^{n_{2}} \mid\|x\| \leqslant a_{1}+a_{2}^{T} x+\right.$ $\left.a_{3}^{T} y, a_{5} \geqslant a_{1}+a_{2}^{T} x+a_{3}^{T} y \geqslant a_{4} \geqslant 0\right\}$ with $a_{1} \in \mathbb{R}, a_{2} \in \mathbb{R}^{n_{1}}, a_{3} \in \mathbb{R}^{n_{2}}$ and $a_{5}>$ $a_{4} \geqslant 0$. Let

$$
\begin{aligned}
& a^{T} \triangleq\left[\begin{array}{lll}
a_{1} & a_{2}^{T} & a_{3}^{T}
\end{array}\right], \\
& b \triangleq a-a_{4} e_{1}, \\
& \bar{b} \triangleq a_{5} e_{1}-a, \\
& e_{1} \triangleq(1,0, \cdots, 0)^{T} \in \mathbb{R}^{1+n_{1}+n_{2}}, \\
& C_{1} \triangleq\left[\begin{array}{lll}
0 & I_{n_{1}} & 0
\end{array}\right], \\
& C_{2} \triangleq\left[\begin{array}{ll}
a & C_{1}^{T}
\end{array}\right] \\
& C_{3} \triangleq a a^{T}-C_{1}^{T} C_{1} .
\end{aligned}
$$


Then we have

$$
\left.\begin{array}{c}
\mathcal{D}_{\mathcal{F}}^{*}=\left\{U=\left[\begin{array}{ccc}
\chi & x^{T} & y^{T} \\
x & X & W^{T} \\
y & W & Y
\end{array}\right] \in \mathcal{S}_{+}^{1+n_{1}+n_{2}} \mid \begin{array}{l}
a^{T} U b \geqslant\left\|C_{1} U b\right\|, e_{1}^{T} U b \geqslant 0, \\
a^{T} U \bar{b} \geqslant\left\|C_{1} U \bar{b}\right\|, e_{1}^{T} U \bar{b} \geqslant 0, \\
b^{T} U \bar{b} \geqslant 0, U \bullet\left(a a^{T}-C_{1}^{T} C_{1}\right) \geqslant 0
\end{array}\right\}, \\
\mathcal{D}_{\mathcal{F}}=\operatorname{cl}\left\{\begin{array}{l}
M-\lambda_{1} C_{3}-\lambda_{2}\left(e_{1} b^{T}+b e_{1}^{T}\right)-\lambda_{3}\left(e_{1} \bar{b}^{T}+\bar{b} e_{1}^{T}\right) \\
-\left(b \psi_{1}^{T} C_{2}^{T}+C_{2} \psi_{1} b^{T}\right) \\
-\left(\bar{b} \psi_{2}^{T} C_{2}^{T}+C_{2} \psi_{2} \bar{b}^{T}\right) \in \mathcal{S}_{+}^{1+n_{1}+n_{2}}, \\
\lambda_{1}, \lambda_{2}, \lambda_{3} \geqslant 0, \psi_{1}, \psi_{2} \in \mathcal{S O C}\left(n_{1}\right)
\end{array}\right.
\end{array}\right\} .
$$

Moreover, the corresponding QCQP and LCoP have the same optimal value.

If there is $(\bar{x}, \bar{y}) \in \mathbb{R}^{n_{1}} \times \mathbb{R}^{n_{2}}$ such that $\|\bar{x}\|<a_{1}+a_{2}^{T} \bar{x}+a_{3}^{T} \bar{y}$ and $a_{5}>a_{1}+$ $a_{2}^{T} \bar{x}+a_{3}^{T} \bar{y}>a_{4}$, then

$$
\mathcal{D}_{\mathcal{F}}=\left\{\begin{array}{l|l}
M \in \mathcal{S}^{1+n_{1}+n_{2}} \mid \begin{array}{c}
M-\lambda_{1} C_{3}-\lambda_{2}\left(e_{1} b^{T}+b e_{1}^{T}\right)-\lambda_{3}\left(e_{1} \bar{b}^{T}+\bar{b} e_{1}^{T}\right) \\
-\left(b \psi_{1}^{T} C_{2}^{T}+C_{2} \psi_{1} b^{T}\right) \\
-\left(\bar{b} \psi_{2}^{T} C_{2}^{T}+C_{2} \psi_{2} \bar{b}^{T}\right) \in \mathcal{S}_{+}^{1+n_{1}+n_{2}}, \\
\lambda_{1}, \lambda_{2}, \lambda_{3} \geqslant 0, \psi_{1}, \psi_{2} \in \mathcal{S O C}\left(n_{1}\right)
\end{array}
\end{array}\right\} .
$$

Moreover, the corresponding problem $L C O D$

$\sup \sigma$

$$
\begin{aligned}
& \text { s.t. } {\left[\begin{array}{cc}
c_{0} & b_{0}^{T} \\
b_{0} & A_{0}
\end{array}\right]-\left[\begin{array}{cc}
\sigma & 0 \\
0 & 0
\end{array}\right]-\lambda_{1} C_{3}-\lambda_{2}\left(e_{1} b^{T}+b e_{1}^{T}\right)-\lambda_{3}\left(e_{1} \bar{b}^{T}+\bar{b} e_{1}^{T}\right) } \\
&-\left(b \psi_{1}^{T} C_{2}^{T}+C_{2} \psi_{1} b^{T}\right)-\left(\bar{b} \psi_{2}^{T} C_{2}^{T}+C_{2} \psi_{2} \bar{b}^{T}\right) \in \mathcal{S}_{+}^{1+n_{1}+n_{2}}, \\
& \sigma \in \mathbb{R}, \lambda_{1}, \lambda_{2}, \lambda_{3} \geqslant 0, \psi_{1}, \psi_{2} \in \mathcal{S O C}\left(n_{1}\right)
\end{aligned}
$$

attains the same optimal value as that of the original QCQP.

Proof Define

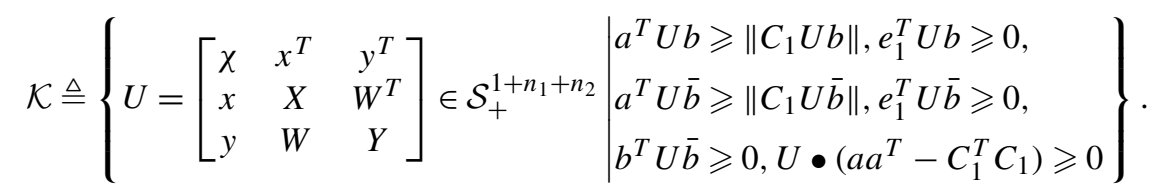

It is clear that $\mathcal{D}_{\mathcal{F}}^{*} \subseteq \mathcal{K}$.

To show $\mathcal{K} \subseteq \mathcal{D}_{\mathcal{F}}^{*}$, it is sufficient to prove that all the extreme points of $\mathcal{K}^{\prime} \triangleq \mathcal{K} \cap$ $\left\{U \in \mathcal{S}^{1+n_{1}+n_{2}} \mid \operatorname{tr} U \leqslant 1\right\}$ belong to $\mathcal{D}_{\mathcal{F}}^{*}$. In other word, for each nonzero extreme point of $\mathcal{K}^{\prime}$, we can find a rank-one decomposition with all elements being in $\mathcal{H}_{\mathcal{F}}$. 
We first prove that

$$
\begin{gathered}
\left\{(t, x, y) \in \mathbb{R}_{+} \times \mathbb{R}^{n_{1}} \times \mathbb{R}^{n_{2}} \mid\|x\| \leqslant a_{1} t+a_{2}^{T} x+a_{3}^{T} y,\right. \\
\left.a_{5} t \geqslant a_{1} t+a_{2}^{T} x+a_{3}^{T} y \geqslant a_{4} t \geqslant 0\right\} \subseteq \mathcal{H}_{\mathcal{F}} .
\end{gathered}
$$

If $t>0$, then $\left[\begin{array}{l}x / t \\ y / t\end{array}\right] \in \mathcal{F}$ and $\left[\begin{array}{l}t \\ x \\ y\end{array}\right] \in \mathcal{H}_{\mathcal{F}}$. If $t=0$, then $x=0$ and $a_{3}^{T} y=0$. Since $\mathcal{F}$ is nonempty, there exists $\left[\begin{array}{l}\bar{x} \\ \bar{y}\end{array}\right] \in \mathcal{F}$. One can verify that $\left[\begin{array}{l}0 \\ 0 \\ y\end{array}\right]+\frac{1}{k}\left[\begin{array}{l}1 \\ \bar{x} \\ \bar{y}\end{array}\right] \in \mathcal{H}_{\mathcal{F}}$. When $k$ goes to infinity, its limit $\left[\begin{array}{l}0 \\ 0 \\ y\end{array}\right] \in \mathcal{H}_{\mathcal{F}}$. Therefore, the above inclusion holds true.

Next, we need to consider five cases for a complete proof: (i) $\chi=0$; (ii) $\chi>0$, $a^{T} U b=\left\|C_{1} U b\right\|$ and $a^{T} U \bar{b}=\left\|C_{1} U \bar{b}\right\|$; (iii) $\chi>0, a^{T} U b>\left\|C_{1} U b\right\|$ and $a^{T} U \bar{b}=$ $\left\|C_{1} U \bar{b}\right\|$; (iv) $\chi>0, a^{T} U b=\left\|C_{1} U b\right\|$ and $a^{T} U \bar{b}>\left\|C_{1} U \bar{b}\right\|$; (v) $\chi>0, a^{T} U b>$ $\left\|C_{1} U b\right\|$ and $a^{T} U \bar{b}>\left\|C_{1} U \bar{b}\right\|$. Let $U^{0}$ be a nonzero extreme point of $\mathcal{K}^{\prime}$.

For case $(i)$ : Corresponding to $U^{0} \neq 0$, we have $\left(x^{0}, y^{0}\right)=0$. Therefore, $a^{T} U^{0} e_{1}=b^{T} U^{0} e_{1}=\bar{b}^{T} U^{0} e_{1}=0$. From $a^{T} U^{0} \bar{b} \geqslant 0$ and $a^{T} U^{0} \bar{b}=a_{5} a^{T} U^{0} e_{1}-$ $a^{T} U^{0} a=-a^{T} U^{0} a \leqslant 0$, we know $U^{0} a=0$. Consequently, $U^{0} b=U^{0} \bar{b}=0$. From $U^{0} \bullet\left(a a^{T}-C_{1}^{T} C_{1}\right)=-\operatorname{tr}\left(C_{1} U^{0} C_{1}^{T}\right)=-\operatorname{tr} X^{0} \leqslant 0$ we have $X^{0}=0$ and $W^{0}=0$. Furthermore, since $U^{0}$ is an extreme point of $\mathcal{K}^{\prime}$, the matrix $Y^{0}$ must be the extreme point of the set

$$
\mathcal{L} \triangleq\left\{Y \in \mathcal{S}_{+}^{n_{2}} \mid \operatorname{tr} Y \leqslant 1, a_{3}^{T} Y a_{3}=0\right\}
$$

and it is a rank-one matrix, i.e., $Y^{0}=y^{0}\left(y^{0}\right)^{T}$ for some $y^{0} \in \mathbb{R}^{n_{2}}$ with $a_{3}^{T} y^{0}=0$. Let $u^{0} \triangleq\left[\begin{array}{c}0 \\ 0 \\ y^{0}\end{array}\right]$, then we have $U^{0}=u^{0}\left(u^{0}\right)^{T}$. Notice that $u^{0} \in \mathcal{H}_{\mathcal{F}}$ and $U^{0} \in \mathcal{D}_{\mathcal{F}}^{*}$.

For Case (ii): When $U^{0} b=0$, we have $e_{1}^{T} U^{0} \bar{b}=e_{1}^{T} U^{0}\left(\left(a_{5}-a_{4}\right) e_{1}-b\right)>0$, which means that $U^{0} \bar{b} \neq 0$. Define $U^{1} \triangleq \frac{U^{0} \bar{b} \bar{b}^{T} U^{0}}{\bar{b}^{T} U^{0} \bar{b}}$ and $U^{2} \triangleq U^{0}-U^{1}$. Then we have

$$
U^{2} \bullet\left(a a^{T}-C_{1}^{T} C_{1}\right)=U^{0} \bullet\left(a a^{T}-C_{1}^{T} C_{1}\right) \geqslant 0 .
$$

We can check all the required conditions in $\mathcal{K}$ to verify that $U^{1}, U^{2} \in \mathcal{K}$. Since $U^{0}$ is an extreme point of $\mathcal{K}^{\prime}$, we have $U^{0}=U^{1}=\frac{U^{0} \bar{b} \bar{b}^{T} U^{0}}{\bar{b}^{T} U^{0} \bar{b}}$. From $U^{0} \bar{b} \in \mathcal{H}_{\mathcal{F}}$, we know $U^{0} \in \mathcal{D}_{\mathcal{F}}^{*}$.

When $U^{0} \bar{b}=0$, similar to the situation of $U^{0} b=0$, we can show that $U^{0}=$ $\frac{U^{0} b b^{T} U^{0}}{b^{T} U^{0} b} \in \mathcal{D}_{\mathcal{F}}^{*}$.

When $U^{0} b \neq 0$ and $U^{0} \bar{b} \neq 0$, define $U^{1} \triangleq \frac{U^{0} b b^{T} U^{0}}{b^{T} U^{0} b}$ and $U^{2} \triangleq U^{0}-U^{1}$. We first consider that $U^{2} \bar{b}=0$. In this case, $U^{0} \bar{b}=\frac{b^{T} U^{0} \bar{b}}{b^{T} U^{0} b} U^{0} b$. Noticing that $U^{2} b=U^{2} \bar{b}=0$ and

$$
U^{2} \bullet\left(a a^{T}-C_{1}^{T} C_{1}\right)=U^{0} \bullet\left(a a^{T}-C_{1}^{T} C_{1}\right) \geqslant 0,
$$

we have $U^{1}, U^{2} \in \mathcal{K}$. Since $U^{0}$ is an extreme point of $\mathcal{K}^{\prime}$, we have $U^{0}=U^{1}=$ $\frac{U^{0} b b^{T} U^{0}}{b^{T} U^{0} b}$. Noticing $U^{0} \bar{b} \in \mathcal{H}_{\mathcal{F}}$, we have $U^{0} \in \mathcal{D}_{\mathcal{F}}^{*}$. Then we consider that $U^{2} \bar{b} \neq 0$. 
In this case, $U^{2} a=\frac{1}{a_{5}-a_{4}}\left(a_{4} U^{2} \bar{b}+a_{5} U^{2} b\right)=\frac{1}{a_{5}-a_{4}} a_{4} U^{2} \bar{b} \neq 0$. Let $U^{3} \triangleq \frac{U^{2} a a^{T} U^{2}}{a^{T} U^{2} a}$ and $U^{4} \triangleq U^{2}-U^{3}=U^{0}-U^{1}-U^{3}$. From Lemma $11, U^{4}$ is positive semidefinite. Therefore,

$$
U^{0} \bullet\left(C_{1}^{T} C_{1}\right)=\left(U^{1}+U^{3}+U^{4}\right) \bullet\left(C_{1}^{T} C_{1}\right) \geqslant\left(U^{1}+U^{3}\right) \bullet\left(C_{1}^{T} C_{1}\right) .
$$

Notice that

$$
\begin{aligned}
& a^{T} U^{2} a\left[\left(U^{1}+U^{3}\right) \bullet\left(C_{1}^{T} C_{1}\right)-a^{T} U^{0} a\right] \\
&=a^{T} U^{2} a\left[\left(\frac{U^{0} b b^{T} U^{0}}{b^{T} U^{0} b}+\frac{\left(U^{0} a-\frac{U^{0} b\left(b^{T} U^{0} a\right)}{b^{T} U^{0} b}\right)\left(U^{0} a-\frac{U^{0} b\left(b^{T} U^{0} a\right)}{b^{T} U^{0} b}\right)^{T}}{a^{T} U^{2} a}\right)\right. \\
&\left.\bullet\left(C_{1}^{T} C_{1}\right)-a^{T} U^{0} a\right] \\
&= \frac{a^{T} U^{2} a\left\|C_{1} U^{0} b\right\|^{2}}{b^{T} U^{0} b}+\left\|C_{1} U^{0} a-\frac{C_{1} U^{0} b\left(b^{T} U^{0} a\right)}{b^{T} U^{0} b}\right\|^{2}-\left(a^{T} U^{2} a\right) a^{T} U^{0} a \\
&= \frac{a^{T} U^{2} a\left(a^{T} U^{0} b\right)^{2}}{b^{T} U^{0} b}+\left\|C_{1} U^{0} a\right\|^{2}-2 \frac{b^{T} U^{0} a}{b^{T} U^{0} b}\left(b^{T} U^{0} C_{1}^{T} C_{1} U^{0} a\right) \\
&+\frac{\left(a^{T} U^{0} b\right)^{4}}{\left(b^{T} U^{0} b\right)^{2}}-\left(a^{T} U^{2} a\right) a^{T} U^{0} a \\
&= 2 \frac{a^{T} U^{0} a\left(a^{T} U^{0} b\right)^{2}}{b^{T} U^{0} b}+\left\|C_{1} U^{0} a\right\|^{2}-2 \frac{b^{T} U^{0} a}{b^{T} U^{0} b}\left(b^{T} U^{0} C_{1}^{T} C_{1} U^{0} a\right)-\left(a^{T} U^{0} a\right)^{2} .
\end{aligned}
$$

Let $\tau_{1} \triangleq \frac{a_{5}}{a_{5}-a_{4}}$ and $\tau_{2} \triangleq \tau_{1}-1$. Then, $a=\tau_{1} b+\tau_{2} \bar{b}$. From $a^{T} U^{0} b=\left\|C_{1} U^{0} b\right\|$ and $a^{T} U^{0} \bar{b}=\left\|C_{1} U^{0} \bar{b}\right\|$, we know

$$
\begin{aligned}
\left\|C_{1} U^{0} a\right\|^{2}= & 2 \tau_{1}\left(b^{T} U^{0} C_{1}^{T} C_{1} U^{0} a\right)+\tau_{2}^{2}\left(a^{T} U^{0} \bar{b}\right)^{2}-\tau_{1} a^{T} U^{0} C_{1}^{T} C_{1} U^{0} b \\
& +\tau_{1} \tau_{2} b^{T} U^{0} C_{1}^{T} C_{1} U^{0} \bar{b} \\
= & 2 \tau_{1}\left(b^{T} U^{0} C_{1}^{T} C_{1} U^{0} a\right)+\tau_{2}^{2}\left(a^{T} U^{0} \bar{b}\right)^{2}-\tau_{1}\left(a^{T} U^{0} b\right) a^{T} U^{0} a \\
& +\tau_{1} \tau_{2}\left(a^{T} U^{0} b\right) a^{T} U^{0} \bar{b}
\end{aligned}
$$

and

$$
\left(a^{T} U^{0} a\right)^{2}=\tau_{1}\left(a^{T} U^{0} b\right) a^{T} U^{0} a+\tau_{2}^{2}\left(a^{T} U^{0} \bar{b}\right)^{2}+\tau_{1} \tau_{2}\left(a^{T} U^{0} b\right) a^{T} U^{0} \bar{b} .
$$

Therefore,

$$
\begin{aligned}
& a^{T} U^{2} a\left[\left(U^{1}+U^{3}\right) \bullet\left(C_{1}^{T} C_{1}\right)-a^{T} U^{0} a\right] \\
& \quad=2\left(\left(a^{T} U^{0} a\right)\left(a^{T} U^{0} b\right)-b^{T} U^{0} C_{1}^{T} C_{1} U^{0} a\right)\left(\frac{a^{T} U^{0} b}{b^{T} U^{0} b}-\tau_{1}\right) \\
& \quad=2\left(\left(a^{T} U^{0} a\right)\left(a^{T} U^{0} b\right)-b^{T} U^{0} C_{1}^{T} C_{1} U^{0} a\right) \frac{\tau_{2} \bar{b}^{T} U^{0} b}{b^{T} U^{0} b} .
\end{aligned}
$$


From $a^{T} U^{0} a=\tau_{1} a^{T} U^{0} b+\tau_{2} a^{T} U^{0} \bar{b}=\tau_{1}\left\|C_{1} U^{0} b\right\|+\tau_{2}\left\|C_{1} U^{0} \bar{b}\right\| \geqslant\left\|C_{1} U^{0} a\right\|$, we have $a^{T} U^{2} a\left[\left(U^{1}+U^{3}\right) \bullet\left(C_{1}^{T} C_{1}\right)-a^{T} U^{0} a\right] \geqslant 0$. Consequently, $U^{0} \bullet\left(C_{1}^{T} C_{1}\right) \geqslant$ $a^{T} U^{0} a$. The equality sign holds if and only if $U^{4} \bullet\left(C_{1}^{T} C_{1}\right)=0$ and the two vectors $\left[\begin{array}{l}a^{T} U^{0} a \\ C_{1} U^{0} a\end{array}\right]$ and $\left[\begin{array}{l}a^{T} U^{0} b \\ C_{1} U^{0} b\end{array}\right]$ are linearly dependent. This also implies that $\left[\begin{array}{l}a^{T} U^{0} \bar{b} \\ C_{1} U^{0} \bar{b}\end{array}\right]$ and $\left[\begin{array}{l}a^{T} U^{0} b \\ C_{1} U^{0} b\end{array}\right]$ are linearly dependent. From this result, we can verify that $U^{1}$ and $U^{2}$ are both in $\mathcal{K}$. Since $U^{0}$ is an extreme point in $\mathcal{K}^{\prime}$, we have $U^{0}=U^{1}=\frac{U^{0} b b^{T} U^{0}}{b^{T} U^{0} b}$. Again, noticing $U^{0} b \in \mathcal{H}_{\mathcal{F}}$, we have $U^{0} \in \mathcal{D}_{\mathcal{F}}^{*}$. This completes the proof of case (ii).

For case (iii): We let $U^{1} \triangleq \lambda U^{0} \bar{b} \bar{b}^{T} U^{0}$ and $U^{2} \triangleq U^{0}-U^{1}$ with $\lambda>0$ being a sufficient small number. One can easily check that $U^{1} \in \mathcal{K}$. Notice that when $\lambda$ is sufficiently small, $U^{2}$ is positive semidefinite. From $a^{T} U^{0} b>\left\|C_{1} U^{0} b\right\|$, we know $\left[\begin{array}{l}a^{T} U^{0} b \\ C_{1} U^{0} b\end{array}\right]$ is an interior point of $\mathcal{S O C}\left(n_{1}\right)$. Therefore, $\left[\begin{array}{l}a^{T} U^{2} b \\ C_{1} U^{2} b\end{array}\right]=\left[\begin{array}{l}a^{T} U^{0} b \\ C_{1} U^{0} b\end{array}\right]-$ $\lambda\left(b^{T} U^{0} \bar{b}\right)\left[\begin{array}{l}a^{T} U^{0} \bar{b} \\ C_{1} U^{0} \bar{b}\end{array}\right] \in \mathcal{S O C}\left(n_{1}\right)$ when $\lambda$ is sufficiently small, i.e., $a^{T} U^{2} b \geqslant\left\|C_{1} U^{2} b\right\|$. We can also see that

$$
\begin{aligned}
& U^{2} \bullet\left(a a^{T}-C_{1}^{T} C_{1}\right)=\left(U^{0}-U^{1}\right) \bullet\left(a a^{T}-C_{1}^{T} C_{1}\right)=U^{0} \bullet\left(a a^{T}-C_{1}^{T} C_{1}\right) \geqslant 0, \\
& b^{T} U^{2} e_{1}=b^{T} U^{0} e_{1}-\lambda\left(b^{T} U^{0} \bar{b}\right) \bar{b}^{T} U^{0} e_{1} \geqslant 0 \quad \text { and } \\
& a^{T} U^{2} \bar{b}=\left(1-\lambda\left(\bar{b}^{T} U^{0} \bar{b}\right)\right) a^{T} U^{0} \bar{b} \geqslant\left\|\left(1-\lambda\left(\bar{b}^{T} U^{0} \bar{b}\right)\right) C_{1} U^{0} \bar{b}\right\|=\left\|C_{1} U^{2} \bar{b}\right\| .
\end{aligned}
$$

Consequently, $U^{2} \in \mathcal{K}$. Remembering that $U^{0}$ is an extreme point of $\mathcal{K}^{\prime}$, we have $U^{0}=\frac{\operatorname{tr} U^{0}}{\operatorname{tr} U^{1}} U^{1}=\frac{\operatorname{tr} U^{0}}{\operatorname{tr} U^{2}} U^{2}$. However, $a^{T} U^{0} b=\frac{\operatorname{tr} U^{0}}{\operatorname{tr} U^{1}} a^{T} U^{1} b=\frac{\operatorname{tr} U^{0}}{\operatorname{tr} U^{1}} \lambda\left(\bar{b}^{T} U^{0} b\right) \times$ $\left(a^{T} U^{0} \bar{b}\right)=\frac{\operatorname{tr} U^{0}}{\operatorname{tr} U^{1}} \lambda\left(\bar{b}^{T} U^{0} b\right)\left\|C_{1} U^{0} \bar{b}\right\|=\frac{\operatorname{tr} U^{0}}{\operatorname{tr} U^{1}}\left\|C_{1} U^{1} b\right\|=\left\|C_{1} U^{0} b\right\|$, which causes a contradiction to the fact of $a^{T} U^{0} b>\left\|C_{1} U^{0} b\right\|$. This means that there is no extreme point of $\mathcal{K}^{\prime}$ to be worried about for case (iii).

For case (iv): The proof is similar to that of case (iii).

For case (v): Let

$$
\mathcal{L}^{\prime} \triangleq\left\{\begin{array}{c|l}
U=\left[\begin{array}{ccc}
x & x^{T} & y^{T} \\
x & x & W^{T} \\
y & W & Y
\end{array}\right], \\
\left(U, S_{1}, S_{2}, s_{1}, s_{2}, s_{3}, s_{4}, s_{5}\right) \in & S_{1}=\operatorname{Arrow}\left(a^{T} U b, C_{1} U b\right), \\
\mathcal{S}_{+}^{1+n_{1}+n_{2}} \times \mathcal{S}_{+}^{1+n_{1}} \times \mathcal{S}_{+}^{1+n_{1}} \times & S_{2}=\operatorname{Arrow}\left(a^{T} U \bar{b}, C_{1} U \bar{b}\right), \\
\mathbb{R}_{+} \times \mathbb{R}_{+} \times \mathbb{R}_{+} \times \mathbb{R}_{+} \times \mathbb{R}_{+} & s_{1}=U \bullet\left(a a^{T}-C_{1}^{T} C_{1}\right), \\
& s_{2}=b^{T} U e_{1}, s_{3}=\bar{b}^{T} U e_{1}, \\
s_{4}=\bar{b}^{T} U b, s_{5}=1-\operatorname{tr} U
\end{array}\right\} .
$$

Since $U^{0}$ is an extreme point of $\mathcal{K}^{\prime}$, the corresponding $\left(U^{0}, S_{1}^{0}, S_{2}^{0}, s_{1}^{0}, s_{2}^{0}, s_{3}^{0}, s_{4}^{0}, s_{5}^{0}\right)$ is an extreme point of $\mathcal{L}^{\prime}$.

From Lemma 9, letting $r_{U} \triangleq \operatorname{rank}\left(U^{0}\right), r_{S_{1}} \triangleq \operatorname{rank}\left(S_{1}^{0}\right), r_{S_{2}} \triangleq \operatorname{rank}\left(S_{2}^{0}\right)$ and $r_{i} \triangleq$ $\operatorname{rank}\left(s_{i}^{0}\right)$ (treat nonnegative number as a matrix of order one), $i=1, \cdots, 5$, we have $r_{U}\left(r_{U}+1\right)+r_{S_{1}}\left(r_{S_{1}}+1\right)+r_{S_{2}}\left(r_{S_{2}}+1\right)+\sum_{i=1}^{5} r_{i}\left(r_{i}+1\right) \leqslant 2\left(n_{1}+1\right)\left(n_{1}+2\right)+10$. 
Under the conditions of case (v), we have $a^{T} U^{0} b>\left\|C_{1} U^{0} b\right\|$ and $a^{T} U^{0} \bar{b}>$ $\left\|C_{1} U^{0} \bar{b}\right\|$. From Lemma 10, $r_{S_{1}}=r_{S_{2}}=1+n_{1}$. Furthermore, $s_{2}^{0}=\frac{1}{a_{5}} b^{T} U^{0}(a+$ $\bar{b})>0$ and $s_{3}^{0}=\frac{1}{a_{5}} \bar{b}^{T} U^{0}(a+\bar{b})>0$. Hence the above inequality becomes

$$
r_{U}\left(r_{U}+1\right)+r_{1}\left(r_{1}+1\right)+r_{4}\left(r_{4}+1\right)+r_{5}\left(r_{5}+1\right) \leqslant 6
$$

If $r_{U}=1$, then one can easily verify that $U^{0} \in \mathcal{D}_{\mathcal{F}}^{*}$. If $r_{U}=2$, we show that $U^{0}$ cannot be an extreme point of $\mathcal{K}^{\prime}$. In this situation, $r_{1}=r_{4}=r_{5}=0$, i.e., $s_{1}^{0}=s_{4}^{0}=$ $s_{5}^{0}=0$. From $a^{T} U^{0} b>0$ and $a^{T} U^{0} \bar{b}>0$, we have $U^{0} b \neq 0$ and $U^{0} \bar{b} \neq 0$. Define $U^{1} \triangleq \frac{U^{0} b b^{T} U^{0}}{b^{T} U^{0} b}$ and $U^{2} \triangleq U^{0}-U^{1}$. From Lemma $11, U^{2}$ is positive semidefinite and $\operatorname{rank}\left(U^{2}\right)=1$. Since $s_{4}^{0}=\bar{b}^{T} U^{0} b=0$, we have $U^{2} \bar{b}=U^{0} \bar{b} \neq 0$. Therefore, $U^{2}=\frac{U^{2} \bar{b} \bar{b}^{T} U^{2}}{\bar{b}^{T} U^{2} \bar{b}}=\frac{U^{0} \bar{b} \bar{b}^{T} U^{0}}{\bar{b}^{T} U^{0} \bar{b}}$. This means $U^{0}=\frac{U^{0} b b^{T} U^{0}}{b^{T} U^{0} b}+\frac{U^{0} \bar{b} \bar{b}^{T} U^{0}}{\bar{b}^{T} U^{0} \bar{b}}$. Notice that $U^{0} \bar{b}$ and $U^{0} b$ are linearly independent. (Otherwise, $0 \neq b^{T} U^{0} b=\tau b^{T} U^{0} \bar{b}=0$ for some $\tau \neq 0$, which causes a contradiction.) One can further verify that $\frac{\operatorname{tr} U^{0}}{\operatorname{tr} U^{1}} U^{1}$ and $\frac{\operatorname{tr} U^{0}}{\operatorname{tr} U^{2}} U^{2}$ are both in $\mathcal{K}^{\prime}$ and $U^{0}$ is a convex combination of these two distinct points. This shows that $U^{0}$ cannot be an extreme point of $\mathcal{K}^{\prime}$.

After checking all the cases, we know $\mathcal{K} \subseteq \mathcal{D}_{\mathcal{F}}^{*}$ and, consequently, $\mathcal{K}=\mathcal{D}_{\mathcal{F}}^{*}$. The proof of the rest part of this theorem is similar to that of Theorem 1. We omit it here.

Remark 6 The proofs in Theorem 1 and Theorem 2 are similar. Here we provide an intuitive but less rigorous discussion about these two theorems. Note that $\bar{b}=$ $a_{5} e_{1}-a$. When $a_{5}=\infty$, then $a_{5} e_{1}$ will dominate $a$ in the definition of $\bar{b}$. Therefore, $\bar{b}$ will be replaced by $e_{1}$ and the computable representation in Theorem 2 degenerates to the one in Theorem 1. However, this approximation will lead to differences in the proofs such as case (i) in each of them.

As in the previous case, when $a_{4}=0$, the results of Theorem 2 can be simplified.

Corollary 2 Given a nonempty set $\mathcal{F}=\left\{(x, y) \in \mathbb{R}^{n_{1}} \times \mathbb{R}^{n_{2}} \mid\|x\| \leqslant a_{1}+a_{2}^{T} x+\right.$ $\left.a_{3}^{T} y, a_{1}+a_{2}^{T} x+a_{3}^{T} y \leqslant a_{5}\right\}$ with $a_{1} \in \mathbb{R}, a_{2} \in \mathbb{R}^{n_{1}}, a_{3} \in \mathbb{R}^{n_{2}}$ and $a_{5} \geqslant 0$. Let

$$
\begin{aligned}
& a^{T} \triangleq\left[\begin{array}{lll}
a_{1} & a_{2}^{T} & a_{3}^{T}
\end{array}\right], \\
& \bar{b} \triangleq a_{5} e_{1}-a, \\
& C_{1} \triangleq\left[\begin{array}{lll}
0 & I_{n_{1}} & 0
\end{array}\right], \\
& e_{1} \triangleq(1,0, \cdots, 0)^{T} \in \mathbb{R}^{1+n_{1}+n_{2}}, \\
& C_{2} \triangleq\left[\begin{array}{ll}
a & C_{1}^{T}
\end{array}\right] \quad \text { and } \\
& C_{3} \triangleq a a^{T}-C_{1}^{T} C_{1} .
\end{aligned}
$$


Then we have

$$
\begin{aligned}
& \mathcal{D}_{\mathcal{F}}^{*}=\left\{U=\left[\begin{array}{ccc}
\chi & x^{T} & y^{T} \\
x & X & W^{T} \\
y & W & Y
\end{array}\right] \in \mathcal{S}_{+}^{1+n_{1}+n_{2}} \mid \begin{array}{l}
a^{T} U \bar{b} \geqslant\left\|C_{1} U \bar{b}\right\|, e_{1}^{T} U \bar{b} \geqslant 0, \\
U \bullet\left(a a^{T}-C_{1}^{T} C_{1}\right) \geqslant 0
\end{array}\right\}, \\
& \mathcal{D}_{\mathcal{F}}=\operatorname{cl}\left\{\begin{array}{l}
M \in \mathcal{S}^{1+n_{1}+n_{2}} \mid \begin{array}{l}
M-\lambda_{1} C_{3}-\lambda_{2}\left(e_{1} \bar{b}^{T}+\bar{b} e_{1}^{T}\right) \\
-\left(\bar{b} \psi^{T} C_{2}^{T}+C_{2} \psi \bar{b}^{T}\right) \in \mathcal{S}_{+}^{1+n_{1}+n_{2}}, \\
\lambda_{1}, \lambda_{2} \geqslant 0, \psi \in \mathcal{S O C}\left(n_{1}\right)
\end{array}
\end{array}\right\} .
\end{aligned}
$$

Moreover, the corresponding QCQP and LCoP have the same optimal value.

If there is $(\bar{x}, \bar{y}) \in \mathbb{R}^{n_{1}} \times \mathbb{R}^{n_{2}}$ such that $\|\bar{x}\|<a_{1}+a_{2}^{T} \bar{x}+a_{3}^{T} \bar{y}$ and $a_{1}+a_{2}^{T} \bar{x}+$ $a_{3}^{T} \bar{y}<a_{5}$, then

$$
\mathcal{D}_{\mathcal{F}}=\left\{\begin{array}{l|l}
M \in \mathcal{S}^{1+n_{1}+n_{2}} & \begin{array}{c}
M-\lambda_{1} C_{3}-\lambda_{2}\left(e_{1} \bar{b}^{T}+\bar{b} e_{1}^{T}\right) \\
-\left(\bar{b} \psi^{T} C_{2}^{T}+C_{2} \psi \bar{b}^{T}\right) \in \mathcal{S}_{+}^{1+n_{1}+n_{2}} \\
\lambda_{1}, \lambda_{2} \geqslant 0, \psi \in \mathcal{S O C}\left(n_{1}\right)
\end{array}
\end{array}\right\} .
$$

Moreover, the corresponding LCoD problem

$$
\begin{array}{ll}
\sup \sigma & \\
\text { s.t. } & {\left[\begin{array}{ll}
c_{0} & b_{0}^{T} \\
b_{0} & A_{0}
\end{array}\right]-\left[\begin{array}{ll}
\sigma & 0 \\
0 & 0
\end{array}\right]-\lambda_{1} C_{3}-\lambda_{2}\left(e_{1} \bar{b}^{T}+\bar{b} e_{1}^{T}\right)} \\
& -\left(\bar{b} \psi^{T} C_{2}^{T}+C_{2} \psi \bar{b}^{T}\right) \in \mathcal{S}_{+}^{1+n_{1}+n_{2}}, \\
& \sigma \in \mathbb{R}, \lambda_{1}, \lambda_{2} \geqslant 0, \psi \in \mathcal{S O C}\left(n_{1}\right)
\end{array}
$$

attains the same optimal value as that of the original $Q C Q P$.

Proof It is sufficient to show that the four constraints in $\mathcal{D}_{\mathcal{F}}^{*}$ of this corollary imply the seven constraints in that of Theorem 2 , when $a_{4}=0$. Let $b \triangleq a-a_{4} e_{1}=a$. From $U \bullet\left(a a^{T}-C_{1}^{T} C_{1}\right) \geqslant 0$ and

$$
\left[\begin{array}{l}
a^{T} \\
C_{1}
\end{array}\right] U\left[\begin{array}{c}
a \\
C_{1}^{T}
\end{array}\right]=\left[\begin{array}{ll}
a^{T} U a & a^{T} U C_{1}^{T} \\
C_{1} U a & C_{1} U C_{1}^{T}
\end{array}\right] \in \mathcal{S}_{+}^{1+n_{1}},
$$

we have $\left(a^{T} U a\right)^{2} \geqslant\left(a^{T} U a\right) \operatorname{tr} C_{1} U C_{1}^{T} \geqslant \operatorname{tr} C_{1} U a a^{T} U C_{1}^{T}=\left\|C_{1} U a\right\|^{2}$, which shows that $a^{T} U b \geqslant\left\|C_{1} U b\right\|$. From $a^{T} U \bar{b} \geqslant 0$ and $e_{1}=\frac{1}{a_{5}}(a+\bar{b})$, we have $b^{T} U e_{1}=\frac{1}{a_{5}} a^{T} U(a+\bar{b}) \geqslant 0$. Moreover, the last constraint is satisfied due to the fact that $\bar{b}^{T} U b=\bar{b}^{T} U a \geqslant 0$. Since all of the seven constraints are satisfied, the rest follows Theorem 2 .

Remark 7 In the literature, a widely used form of the second-order cone constraint is $c^{T} x+d \geqslant\|A x+b\|$, in which $c \in \mathbb{R}^{n}, d \in \mathbb{R}, A \in \mathbb{R}^{m \times n}$ and $b \in \mathbb{R}^{m}$. In this case, 
the domain $\mathcal{F}$ can be equivalently written as $\mathcal{F} \triangleq\left\{\left(x, y_{0}, y\right) \in \mathbb{R}^{n} \times \mathbb{R} \times \mathbb{R}^{m} \mid y_{0} \geqslant\right.$ $\left.\|y\|, A x+b=y, c^{T} x+d=y_{0}\right\}$. From Lemma 8 and Corollary 1 , we have

$$
\mathcal{D}_{\mathcal{F}}^{*}=\left\{\begin{array}{l|l}
U=\left[\begin{array}{cc}
\chi & w^{T} \\
w & W
\end{array}\right] \in \mathcal{S}_{+}^{2+m+n} & \begin{array}{l}
w=\left[\begin{array}{c}
x \\
y_{0} \\
y
\end{array}\right], W=\left[\begin{array}{ccc}
X & W_{x y_{0}}^{T} & W_{x y}^{T} \\
W_{x y_{0}} & Y_{0} & W_{y_{0} y}^{T} \\
W_{x y} & W_{y_{0} y} & Y
\end{array}\right], \\
y_{0} \geqslant\|y\|, Y_{0} \geqslant \operatorname{tr} Y, B w=\left[\begin{array}{c}
-b \chi \\
-d \chi
\end{array}\right], \\
\operatorname{diag}\left(B W B^{T}\right)=\left[\begin{array}{c}
\chi(b \circ b) \\
\chi d^{2}
\end{array}\right]
\end{array}
\end{array}\right\}
$$

where $B \triangleq\left[\begin{array}{ccc}A & 0 & -I_{m} \\ c^{T} & -1 & 0\end{array}\right]$. Therefore, a computable representation is also available for the domain defined by the second-order cone constraint in the widely used form.

Similarly, one can obtain the computable representation of $c^{T} x+d \geqslant\|A x+b\|$ with $l \leqslant c^{T} x+d \leqslant u$, in which $c \in \mathbb{R}^{n}, d, l, u \in \mathbb{R}, A \in \mathbb{R}^{m \times n}$ and $b \in \mathbb{R}^{m}$.

Remark 8 According to Lemma 6, from Theorems 1, 2 and Corollary 2, a bigger set $\mathcal{F}=\left\{(x, y) \in \mathbb{R}^{n_{1}} \times \mathbb{R}^{n_{2}} \mid x^{T} x \leqslant\left(a_{1}+a_{2}^{T} x+a_{3}^{T} y\right)^{2}, a_{4} \leqslant a_{1}+a_{2}^{T} x+a_{3}^{T} y \leqslant a_{5}\right\}$ with $a_{4}, a_{5} \in \mathbb{R}$ can be treated as the union of several sets discussed in the above theorems. Consequently, this set $\mathcal{F}$ also has a computable representation.

\section{Concluding Remarks}

In this paper, we have developed an exact computable representation of the QCQP problem whose feasible domain is defined by one second-order cone constraint and two special linear constraints. In each case, the representation involves a linear conic programming problem with linear, second-order cone and semidefinite constraints. We have shown that finding an optimal extreme solution to such a linear conic program can lead to an optimal solution to the original QCQP problem. In particular, we now know that the problem of optimizing a nonconvex quadratic function subject to one general second-order cone constraint is computable. We expect the results obtained will further advance the study of copositive programming problems.

Acknowledgements The authors would like to thank the anonymous reviewers for their valuable comments and suggestions to improve the quality of the paper.

\section{References}

[1] Burer, S.: On the copositive representation of binary and continuous nonconvex quadratic programs. Math. Program. 120, 479-495 (2009)

[2] Burer, S., Anstreicher, K.M.: Second-order cone constraints for extended trust-region subproblems. SIAM J. Optim. (2012). www.optimizationonline.org/DBHTML/2011/03/2957.html

[3] Burer, S.: Copositive programming. In: Anjos, M.F., Lasserre, J.B. (eds.) Handbook on Semidefinite, Conic and Polynomial Optimization. Springer, Berlin (2011)

[4] Burer, S., Dong, H.: Representing quadratically constrained quadratic programs as generalized copositive programs. Oper. Res. Lett. 40, 203-206 (2011) 
[5] Eichfelder, G., Povh, J.: On the set-semidefinite representation of non-convex quadratic programs with cone constraints. Croat. Oper. Res. Rev. 1, 26-39 (2011)

[6] Eichfelder, G., Povh, J.: On the set-semidefinite representation of nonconvex quadratic programs over arbitrary feasible sets. Optim. Lett. (2012). doi:10.1007/s11590-012-0450-3

[7] Kim, S., Kojima, M.: Exact solutions of some nonconvex quadratic optimization problems via SDP and SOCP relaxations. Comput. Optim. Appl. 26, 143-154 (2003)

[8] Kojima, M., Kim, S., Waki, H.: A general framework for convex relaxation of polynomial optimization problems over cones. J. Oper. Res. Soc. Jpn. 46, 125-144 (2003)

[9] Nemirovskii, A., Scheinberg, K.: Extension of Karmarkar's algorithm onto convex quadratically constrained quadratic problems. Math. Program. 72, 273-289 (1996)

[10] Pardalos, P.M., Vavasis, S.A.: Quadratic programming with one negative eigenvalue is NP-Hard. J. Glob. Optim. 1, 15-22 (1991)

[11] Pataki, G.: On the rank of extreme matrices in semidefinite programs and the multiplicity of optimal eigenvalues. Math. Oper. Res. 23, 339-358 (1998)

[12] Rockafellar, R.T.: Convex Analysis. Princeton University Press, Princeton (1972)

[13] Sturm, J.F., Zhang, S.: On cones of nonnegative quadratic functions. Math. Oper. Res. 28, 246-267 (2003)

[14] Ye, Y., Zhang, S.: New results on quadratic minimization. SIAM J. Optim. 14, 245-267 (2003) 